\title{
Monomial Ideals, Binomial Ideals, Polynomial Ideals
}

\author{
BERNARD TEISSIER
}

\begin{abstract}
These lectures provide a glimpse of the applications of toric geometry to singularity theory. They illustrate some ideas and results of commutative algebra by showing the form which they take for very simple ideals of polynomial rings: monomial or binomial ideals, which can be understood combinatorially. Some combinatorial facts are the expression for monomial or binomial ideals of general results of commutative algebra or algebraic geometry such as resolution of singularities or the BriançonSkoda theorem. In the opposite direction, there are methods that allow one to prove results about fairly general ideals by continuously specializing them to monomial or binomial ideals.
\end{abstract}

\section{Contents}

1. Introduction 211

2. Strong Principalization of Monomial Ideals by Toric Maps 213

3. The Integral Closure of Ideals 218

4. The Monomial Briançon-Skoda Theorem 220

5. Polynomial Ideals and Nondegeneracy 222

6. Resolution of Binomial Varieties 229

7. Resolution of Singularities of Branches 233

Appendix: Multiplicities, Volumes and Nondegeneracy 237

References

\section{Introduction}

Let $k$ be a field. We denote by $k\left[u_{1}, \ldots, u_{d}\right]$ the polynomial ring in $d$ variables, and by $k \llbracket u_{1}, \ldots, u_{d} \rrbracket$ the power series ring.

If $d=1$, given two monomials $u^{m}, u^{n}$, one divides the other, so that if $m>n$, say, a binomial $u^{m}-\lambda u^{n}=u^{n}\left(u^{m-n}-\lambda\right)$ with $\lambda \in k^{*}$ is, viewed now in $k \llbracket u \rrbracket$, a monomial times a unit. For the same reason any series $\sum_{i} f_{i} u^{i} \in k \llbracket u \rrbracket$ is the product of a monomial $u^{n}, n \geq 0$, by a unit of $k \llbracket u \rrbracket$. Staying in $k[u]$, we can 
also view our binomial as the product of a monomial and a cyclic polynomial $u^{m-n}-\lambda$.

For $d=2$, working in $k \llbracket u_{1}, u_{2} \rrbracket$, we meet a serious difficulty: a series in two variables does not necessarily have a dominant term (a term that divides all others). The simplest example is the binomial $u_{1}^{a}-c u_{2}^{b}$ with $c \in k^{*}$. As we shall see, if we allow enough transformations, this is essentially the only example in dimension 2. So the behavior of a series $f\left(u_{1}, u_{2}\right)$ near the origin does not reduce to that of the product of a monomial $u_{1}^{a} u_{2}^{b}$ by a unit.

In general, for $d>1$ and given $f\left(u_{1}, \ldots, u_{d}\right) \in k \llbracket u_{1}, \ldots, u_{d} \rrbracket$, say $f=$ $\sum_{m} f_{m} u^{m}$, where $m \in \mathbf{Z}_{\geq 0}^{d}$ and $u^{m}=u_{1}^{m_{1}} \ldots u_{d}^{m_{d}}$, we can try to measure how far $f$ is from a monomial times a unit by considering the ideal of $k \llbracket u_{1}, \ldots, u_{d} \rrbracket$ or $k\left[u_{1}, \ldots, u_{d}\right]$ generated by the monomials $\left\{u^{m}: f_{m} \neq 0\right\}$ that actually appear in $f$. Since both rings are noetherian, this ideal is finitely generated in both cases, and we are faced with the following problem:

Problem. Given an ideal generated by finitely many monomials (a monomial ideal) in $k \llbracket u_{1}, \ldots, u_{d} \rrbracket$ or $k\left[u_{1}, \ldots, u_{d}\right]$, study how far it is from being principal.

We shall also meet a property of finitely generated ideals that is stronger than principality, namely that given any pair of generators, one divides the other. This implies principality (exercise), but is stronger in general: take an ideal in a principal ideal domain such as $\mathbf{Z}$, or a nonmonomial ideal in $k[u]$. I shall call this property strong principality. Integral domains in which every finitely generated ideal is strongly principal are known as valuation rings. Most are not noetherian.

Here we reach a bifurcation point in methodology:

- One approach is to generalize the notion of divisibility by studying all linear relations, with coefficients in the ambient ring, between our monomials. This leads to the construction of syzygies for the generators of our monomial ideal $\mathscr{M}$, or free resolutions for the quotient of the ambient ring by $\mathscr{M}$. There are many beautiful results in this direction; see [Eisenbud and Sidman 2004] in this volume and [Sturmfels 1996]. One is also led to try and compare monomials using monomial orders to produce Gröbner bases, since as soon as the ideal is not principal, deciding whether a given element belongs to it becomes arduous in general.

- Another approach is to try and force the ideal $\mathscr{M}$ to become principal after a change of variables. This is the subject of the next section. 


\section{Strong Principalization of Monomial Ideals by Toric Maps}

In order to make a monomial ideal principal by changes of variables, the first thing to try is changes of variables that transform monomials into monomials, that is, which are themselves described by monomial functions:

$$
\begin{aligned}
& u_{1}=y_{1}{ }^{a_{1}^{1}} \cdots y_{d}{ }^{a_{1}^{d}}, \\
& u_{2}=y_{1}{ }^{a_{2}^{1}} \cdots y_{d}^{a_{2}^{d}}, \\
& \cdots \cdots \cdots \cdots \cdot \cdots \cdot y_{d}^{a_{d}^{d}}, \\
& u_{d}=y_{1}{ }^{a_{d}^{1}} \cdots \cdots
\end{aligned}
$$

where we can consider the exponents of $y_{i}$ appearing in the expressions of $u_{1}, \ldots, u_{d}$ as the coordinates of a vector $a^{i}$ with integral coordinates. These expressions decribe a monomial, or toric, map of $d$-dimensional affine spaces

$$
\pi\left(a^{1}, \ldots, a^{d}\right): \mathbf{A}^{d}(k) \rightarrow \mathbf{A}^{d}(k)
$$

in the coordinates $\left(y_{i}\right)$ for the first affine space and $\left(u_{i}\right)$ for the second.

If we compute the effect of the change of variables on a monomial $u^{m}$, we see that

$$
u^{m} \mapsto y_{1}^{\left\langle a^{1}, m\right\rangle} \ldots y_{d}^{\left\langle a^{d}, m\right\rangle} .
$$

Exercise. Show that the degree of the fraction field extension $k\left(u_{1}, \ldots, u_{d}\right) \rightarrow$ $k\left(y_{1}, \ldots, y_{d}\right)$ determined by $\pi\left(a^{1}, \ldots, a^{d}\right)$ is the absolute value of the determinant of the vectors $\left(a^{1}, \ldots, a^{d}\right)$. In particular, it is equal to one - that is, our map $\pi\left(a^{1}, \ldots, a^{d}\right)$ is birational - if and only if the determinant of the vectors $\left(a^{1}, \ldots, a^{d}\right)$ is \pm 1 , that is, $\left(a^{1}, \ldots, a^{d}\right)$ is a basis of the integral lattice $\mathbf{Z}^{d}$.

In view of the form of the transformation on monomials by our change of variables, it makes sense to introduce a copy of $\mathbf{Z}^{d}$ where the exponents of our monomials dwell, and which we will denote by $M$, and a copy of $\mathbf{Z}^{d}$ in which our vectors $a^{j}$ dwell, which we will call the weight space and denote by $W$. The lattices $M$ and $W$ are dual and we consider $W$ as the integral lattice of the vector space $\check{\mathbf{R}}^{d}$ dual to the vector space $\mathbf{R}^{d}$ in which our monomial exponents live. In this manner, we think of $m \mapsto\left\langle a^{i}, m\right\rangle$ as the linear form on $M$ corresponding to $a^{i} \in W$.

Given two monomials $u^{m}$ and $u^{n}$, the necessary and sufficient condition for the transform of $u^{n}$ to divide the transform of $u^{m}$ in $k\left[y_{1}, \ldots, y_{d}\right]$ is that $\left\langle a^{i}, m\right\rangle \geq$ $\left\langle a^{i}, n\right\rangle$ for all $i$ with $1 \leq i \leq d$. If we read this as $\left\langle a^{i}, m-n\right\rangle \geq 0$ for all $i$, $1 \leq i \leq d$, and seek a symmetric formulation, we are led to introduce the rational hyperplane $H_{m-n}$ in $\check{\mathbf{R}}^{d}$ dual to the vector $m-n \in M$, and obtain the following elementary but fundamental fact, where the transform of a monomial is just its composition with the map $\pi\left(a^{1}, \ldots, a^{d}\right)$ in the coordinates $\left(y_{1}, \ldots, y_{d}\right)$ :

LEMMA 2.1. A necessary and sufficient condition for the transform of one of the monomials $u^{m}, u^{n}$ by the map $\pi\left(a^{1}, \ldots, a^{d}\right)$ to divide the transform of the other 
in $k\left[y_{1}, \ldots, y_{d}\right]$ is that all the vectors $a^{j}$ lie on the same side of the hyperplane $H_{m-n}$ in $\check{\mathbf{R}}_{\geq 0}^{d}$.

The condition is nonvacuous if and only if one of the monomials $u^{m}, u^{n}$ does not already divide the other in $k\left[u_{1}, \ldots, u_{d}\right]$, because to say that such divisibility does not occur is to say that the equation of the hyperplane $H_{m-n}$ does not have all its coefficients of the same sign, and therefore separates into two regions the first quadrant $\check{\mathbf{R}}_{\geq 0}^{d}$ where our vectors $a^{j}$ live.

To force one monomial to divide the other in the affine space $\mathbf{A}^{d}(k)$ with coordinates $\left(y_{i}\right)$ is nice, but not terribly useful, since it provides information on the original monomials only in the image of the map $\pi\left(a^{1}, \ldots, a^{d}\right)$ in the affine space $\mathbf{A}^{d}(k)$ with coordinates $\left(u_{i}\right)$, which is a constructible subset different from $\mathbf{A}^{d}(k)$. It is much more useful to find a proper and birational (hence surjective) map $\pi: Z \rightarrow \mathbf{A}^{d}(k)$ of algebraic varieties over $k$ such that the compositions with $\pi$ of our monomials generate a sheaf of ideals in $Z$ which is locally principal; if you prefer, $Z$ should be covered by affine charts $U$ such that if our monomial ideal $\mathscr{M}$ is generated by $u^{m^{1}}, \ldots, u^{m^{q}}$, the ideal $\left.\left(u^{m^{1}} \circ \pi, \ldots, u^{m^{q}} \circ \pi\right)\right|_{U}$ is principal or strongly principal.

Toric geometry provides a way to do this. To set the stage, we need a few definitions (see [Ewald 1996]):

A cone $\sigma$ in $\mathbf{R}^{d}$ (or $\check{\mathbf{R}}^{d}$ ) is a set closed under multiplication by nonnegative numbers. A cone is strictly convex if it contains no positive-dimensional vector subspace. Cones contained in the first quadrant are strictly convex. The convex dual of $\sigma$ is the set

$$
\check{\sigma}=\left\{m \in \mathbf{R}^{d}:\langle m, a\rangle \geq 0 \text { for all } a \in \sigma\right\} .
$$

This is also a cone. A cone is strictly convex if and only if its convex dual has nonempty interior.

A rational convex cone is one bounded by finitely many hyperplanes whose equations have rational (or equivalently, integral) coefficients. An equivalent definition is that a rational convex cone is the cone positively generated by finitely many vectors with integral coordinates.

A rational fan with support $\check{\mathbf{R}}_{\geq 0}^{d}$ is a finite collection $\Sigma$ of rational strictly convex cones $\left(\sigma_{\alpha}\right)_{\alpha \in A}$ with the following properties:

(1) The union of all the $\left(\sigma_{\alpha}\right)_{\alpha \in A}$ is the closed first quadrant $\check{\mathbf{R}}_{\geq 0}^{d}$ of $\check{\mathbf{R}}^{d}$.

(2) Each face of a $\sigma_{\alpha} \in \Sigma$ is in $\Sigma$; in particular $\{0\} \in \Sigma$.

(3) Each intersection $\sigma_{\alpha} \cap \sigma_{\beta}$ is a face of $\sigma_{\alpha}$ and of $\sigma_{\beta}$.

In general, the support of a fan $\Sigma$ is defined as $\bigcup_{\alpha \in A} \sigma_{\alpha}$.

A fan is regular if each of its $k$-dimensional cones is generated by $k$ integral vectors (a simplicial cone) that form part of a basis of the integral lattice. If $k=d$ this means that their determinant is \pm 1 . 
If we go back to our monomial map, assuming that the determinant of the vectors $\left(a^{1}, \ldots, a^{d}\right)$ is \pm 1 , we can express the $y_{j}$ as monomials in the $u_{i}$; the matrix of exponents will then be the inverse of the matrix $\left(a^{1}, \ldots, a^{d}\right)$, and will have some negative entries. Monomials with possibly negative exponents will be called Laurent monomials here.

If $\sigma=\left\langle a^{1}, \ldots, a^{d}\right\rangle$, the cone positively generated by the vectors $a^{1} \ldots, a^{d}$, then the monomials in $y_{1}, \ldots, y_{d}$, viewed as Laurent monomials in $u_{1}, \ldots u_{d}$ via the expression of the $y_{j}$ as Laurent monomials in the $u_{i}$, correspond to the integral points of the convex dual cone of $\sigma$, that is, those points $m \in \mathbf{Z}^{d}$ such that $\left\langle a^{i}, m\right\rangle \geq 0$ for all $1 \leq i \leq d$. So we can identify the polynomial algebra $k\left[y_{1}, \ldots, y_{d}\right]$ with the algebra $k[\check{\sigma} \cap M]$ of the semigroup $\check{\sigma} \cap M$ with coefficients in $k$. Since $\sigma$ is contained in the first quadrant of $\check{\mathbf{R}}^{d}$, its convex dual $\check{\sigma}$ contains the first quadrant of $\mathbf{R}^{d}$, so we have a graded inclusion of algebras

$$
k\left[\mathbf{R}_{\geq 0}^{d} \cap M\right]=k\left[u_{1}, \ldots, u_{d}\right] \subset k[\check{\sigma} \cap M]=k\left[y_{1}, \ldots, y_{d}\right],
$$

the inclusion being described by sending each variable $u_{i}$ to a monomial in $y_{1}, \ldots, y_{d}$ as we did in the beginning.

This slightly more abstract formulation has the following use: Given a fan in $\check{\mathbf{R}}^{d}$, to each of its cones $\sigma$ we can associate the algebra $k[\check{\sigma} \cap M]$, even if the strictly convex cone $\sigma$ is not generated by $d$ vectors with determinant \pm 1 .

By a lemma of Gordan [Kempf et al. 1973], the algebra $k[\check{\sigma} \cap M]$ is finitely generated, so it corresponds to an affine algebraic variety $X_{\sigma}=\operatorname{Spec} k[\check{\sigma} \cap M]$. This variety is a $d$-dimensional affine space if and only if the cone $\check{\sigma}$ (or $\sigma$ ) is $d$-dimensional and generated by vectors that form a basis of the integral lattice of $\check{\mathbf{R}}^{d}$. It is, however, always normal and has rational singularities only [Kempf et al. 1973]; moreover it is rational, which means that the field of fractions of $k[\check{\sigma} \cap M]$ is $k\left(u_{1}, \ldots, u_{d}\right)$.

If two cones $\sigma_{\alpha}$ and $\sigma_{\beta}$ have a common face $\tau_{\alpha \beta}$, the affine varieties $X_{\sigma_{\alpha}}$ and $X_{\sigma_{\beta}}$ can be glued up along the open set corresponding to the shared $X_{\tau_{\alpha \beta}}$. By this process, the fan $\Sigma$ gives rise to an algebraic variety $Z(\Sigma)$ proper over $\mathbf{A}^{d}(k)$ :

$$
\pi(\Sigma): Z(\Sigma) \rightarrow \mathbf{A}^{d}(k) .
$$

The variety $Z(\Sigma)$ is covered by affine charts corresponding to the $d$-dimensional cones $\sigma$ of $\Sigma$, and in each of these charts the map $\pi(\Sigma)$ corresponds to the inclusion of algebras $k\left[u_{1}, \ldots, u_{d}\right] \subset k[\check{\sigma} \cap M]$. If $\sigma$ is generated by $d$ vectors forming a basis of the integral lattice (determinant \pm 1 ), the latter algebra is a polynomial ring $k\left[y_{1}, \ldots, y_{d}\right]$ and the inclusion is given by the monomial expression we started from.

Definition. A convex polyhedral cone $\sigma$ is compatible with a convex polyhedral cone $\sigma^{\prime}$ if $\sigma \cap \sigma^{\prime}$ is a face of each. A fan is compatible with a polyhedral cone if each of its cones is.

Remember that $\{0\}$ is a face of every strictly convex cone. 
Lemma 2.2. Given two monomials $u^{m}, u^{n}$, if we can find a fan $\Sigma$ compatible with the hyperplane $H_{m-n}$ in the weight space, then in each chart of $Z(\Sigma)$ the transform of one of our monomials will divide the other.

Proof. This follows from Lemma 2.1.

ExAmple. In dimension $d=2$, let's try to make one of the two monomials $\left(u_{1}, u_{2}\right)$ divide the other after a monomial transformation. The hyperplane in the weight space is $w_{1}=w_{2}$; its intersection with the first quadrant defines a fan whose cones are $\sigma_{1}$ generated by $a^{1}=(0,1), a^{2}=(1,1)$ and $\sigma_{2}$ generated by $b^{1}=(1,1), b^{2}=(0,1)$, together with and their faces. The semigroup of integral points of $\check{\sigma}_{1} \cap M$ is generated by $(1,0),(-1,1)$, which correspond respectively to the monomials $y_{1}=u_{1}, y_{2}=u_{1}^{-1} u_{2}$. The semigroup of integral points of $\check{\sigma}_{2} \cap M$ is generated by $(0,1),(1,-1)$, which correspond to $y_{2}^{\prime}=u_{2}, y_{1}^{\prime}=u_{1} u_{2}^{-1}$. There is a natural isomorphism of the open sets where $u_{1} \neq 0$ and $u_{2} \neq 0$, and gluing gives the two-dimensional subvariety of $\mathbf{A}^{2}(k) \times \mathbf{P}^{1}(k)$ defined by $t_{2} u_{1}-t_{1} u_{2}=0$, where $\left(t_{1}: t_{2}\right)$ are the homogeneous coordinates on $\mathbf{P}^{1}(k)$, with its natural projection to $\mathbf{A}^{2}(k)$ : it is the blowing-up of the origin in $\mathbf{A}^{2}(k)$.
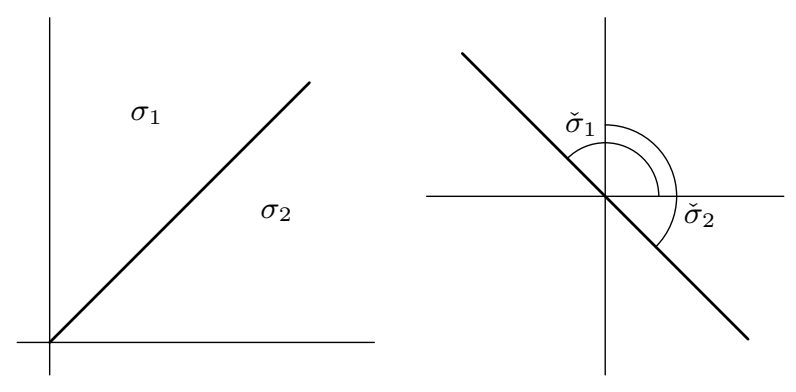

Now if we have a finite number of distinct monomials $\neq 1$, say $u^{m^{1}}, \ldots, u^{m^{q}}$, and if we can find a fan $\Sigma$ with support $\check{\mathbf{R}}_{\geq 0}^{d}$ and compatible with all the hyperplanes $H_{m^{s}-m^{t}}, 1 \leq s, t \leq q, s \neq t$, this will give us an algebraic (toric) variety $Z(\Sigma)$, possibly singular and endowed with a proper surjective map $\pi(\Sigma): Z(\Sigma) \rightarrow$ $\mathbf{A}^{d}(k)$ such that the pullback by $\pi(\Sigma)$ of the ideal $\mathscr{M}$ generated by our monomials is strongly principal in each chart. Properness and surjectivity are ensured (see [Kempf et al. 1973]) by the fact that the support of $\Sigma$ is $\mathbf{R}_{\geq 0}^{d}$.

Our collection of hyperplanes $H_{m^{s}-m^{t}}, 1 \leq s, t \leq q, s \neq t$ through the origin in fact defines a fan $\Sigma_{0}(F)$ that depends only upon the finite set $F=\left\{m^{1}, \ldots, m^{q}\right\}$ of elements of $\mathbf{Z}^{d}$ : take as cones the closures of the connected components of the complement in $\check{\mathbf{R}}_{\geq 0}^{d}$ of the union of all the hyperplanes. They are strictly convex rational cones because they lie in the first quadrant and are bounded by hyperplanes whose equations have integral coefficients. Add all the faces of these cones, and we have a fan, of course not regular in general. To say that a monomial ideal generated by monomials in the generators of the algebra $k[\check{\sigma} \cap M]$ is locally strongly principal is not nearly as useful when these generators do not 
form a system of coordinates as when they do. However, note that we first make our ideal $\mathscr{M}$ locally strongly principal via the map $\pi\left(\Sigma_{0}\right): Z\left(\Sigma_{0}\right) \rightarrow \mathbf{A}^{d}(k)$, and then resolve the singularities of $Z\left(\Sigma_{0}\right)$ using a toric map.

The second step corresponds to a refinement of $\Sigma_{0}$ into a regular fan $\Sigma$, where refinement means that each cone of the second fan in contained in a cone of the original.

This is always possible in view of a result of Kempf, Knudsen, Mumford and St. Donat:

Theorem 2.3 [Kempf et al. 1973]. A rational fan can always be refined into a regular fan.

From this follows:

Theorem 2.4. Let $k$ be a field. Given a monomial ideal $\mathscr{M}$ in $k\left[u_{1}, \ldots, u_{d}\right]$, there exists a fan $\Sigma_{0}$ with support $\check{\mathbf{R}}_{\geq 0}^{d}$ such that, given any regular refinement $\Sigma$ of $\Sigma_{0}$, the associated proper birational toric map of nonsingular toric varieties

$$
\pi(\Sigma): Z(\Sigma) \rightarrow \mathbf{A}^{d}(k)
$$

has the property that the transform of $\mathscr{M}$ is strongly principal in each chart.

REMARK. By construction, for each chart $Z(\sigma)$ of $Z(\Sigma)$ there is an element of $\mathscr{M}$ whose transform generates the ideal $\mathscr{M}_{Z(\sigma)}$. This element cannot be the same for all charts unless $\mathscr{M}$ is already principal.

To see this, assume that there is a monomial $u^{n}$ whose transform generates $\mathscr{M} \mathscr{O}_{Z(\Sigma)}$ in every chart. This means that every simplicial cone $\sigma$ of our fan with support $\check{\mathbf{R}}_{\geq 0}^{d}$ is on the positive side of all the hyperplanes $H_{m-n}$ for all other monomials $u^{m}$ generating $\mathscr{M}$. But this is possible only if none of these hyperplanes meets the positive quadrant outside $\{0\}$, which means that $u^{n}$ divides all the other $u^{m}$.

Remark (Strong PRincipalization And Blowing-up). Given a finitely generated ideal $I$ in a commutative integral domain $R$, there is a proper birational map $\pi: B(I) \rightarrow$ Spec $R$, unique up to unique isomorphism, with the property that the ideal sheaf $I \mathscr{O}_{B(I)}$ generated by the compositions with $\pi$ of the elements of $I$ is locally principal and generated by a nonzero divisor (that is, it's an invertible ideal), and that any map $W \rightarrow \operatorname{Spec} R$ with the same property factors uniquely through $\pi$. The map $\pi$ is called the blowing-up of $I$ in $R$, or in $\operatorname{Spec} R$.

The blowing-up is independent of the choice of generators of $I$. Since a product of ideals is invertible if and only if each ideal is, for $I=\left(f_{1}, \ldots, f_{s}\right) R$ the blowingup in Spec $R$ of the ideal $J=\prod_{i<j}\left(f_{i}, f_{j}\right) R$ will make $I$ strongly principal.

If $I$ is a monomial ideal in $k\left[u_{1}, \ldots, u_{d}\right]$, according to [Kempf et al. 1973], the blowing-up of $I$ followed by normalization is the equivariant map associated to the fan dual to the Newton polyhedron of $I$. (The Newton polyhedron is defined in the Appendix.) The reader is encouraged to check that the fan just mentioned 
admits the fan $\Sigma_{0}$ introduced above as a refinement, illustrating the general fact that a strong principalization map factors through the blowing-up.

Strong principalization is stressed in these lectures because it is directly linked with the resolution of singularities of binomial ideals explained in Section 6 .

ExERCISE. Check that one can in all statements and proofs in this section replace the positive quadrant of $\check{\mathbf{R}}^{d}$ by any strictly convex rational cone $\sigma_{0} \subset \check{\mathbf{R}}^{d}$. The affine space $\mathbf{A}^{d}(k)$ is then replaced by the affine toric variety $X_{\sigma_{0}}$.

\section{The Integral Closure of Ideals}

Given a finite set $F=\left\{m^{1}, \ldots, m^{q}\right\}$ of elements of $\mathbf{Z}^{d}$, define its support function as the function $\mathrm{h}_{F}: \check{\mathbf{R}}^{d} \rightarrow \mathbf{R}$ defined by

$$
\mathrm{h}_{F}(\ell)=\min _{1 \leq s \leq q} \ell\left(m^{s}\right) \text {. }
$$

For reasons that will become apparent, I denote the convex hull of $F$ by $\bar{F}$. It is a classical result that

$$
\bar{F}=\left\{n \in \mathbf{R}^{d}: \ell(n) \geq \mathrm{h}_{F}(\ell) \text { for all } \ell \in \check{\mathbf{R}}^{d}\right\} ;
$$

in words, the convex hull of a set is the intersection of the half-spaces containing that set (or, as often stated in books on convexity, a convex set is the intersection of the half-spaces determined by its support hyperplanes). The proof of this statement also shows that the "positive convex hull" is defined by the same inequalities, restricted to the linear forms lying in the positive quadrant of $\check{\mathbf{R}}^{d}$ :

$$
\overline{\bigcup_{1 \leq s \leq q}\left(m^{s}+\mathbf{R}_{\geq 0}^{d}\right)}=\left\{n \in \mathbf{R}^{d}: \ell(n) \geq \mathrm{h}_{F}(\ell) \text { for all } \ell \in \check{\mathbf{R}}_{\geq 0}^{d}\right\} .
$$

LEMMA 3.1. The support function $\mathrm{h}_{F}$ is linear in each cone of the fan $\Sigma_{0}(F)$ introduced in Section 2.

ProOF. This follows directly from the definitions.

Choose a strongly principalizing map $\pi(\Sigma): Z(\Sigma) \rightarrow \mathbf{A}^{d}(k)$ with $\Sigma$ a refinement of $\Sigma_{0}(F)$, as in Theorem 2.4. Then $Z(\Sigma)$ is normal by [Kempf et al. 1973] (it is regular if $\Sigma$ is regular), and $\pi(\Sigma)$ is proper and birational. Let $u^{n}$ be a monomial in $k\left[u_{1}, \ldots, u_{d}\right]$. Given a chart $X_{\sigma}$ of $Z(\Sigma)$, corresponding to $\sigma \in \Sigma$, a necessary and sufficient condition for $u^{n} \circ \pi(\Sigma)$ to belong in $k[\check{\sigma} \cap M]$ to the ideal generated by the transforms of the generators of $\mathscr{M}$ is that we have $\ell(n) \geq \mathrm{h}_{F}(\ell)$ for all $\ell \in \sigma$ : by Lemma 3.1, we have for some $t \in\{1, \ldots, q\}$ that $\mathrm{h}_{F}(\ell)=\ell\left(m^{t}\right)$ for all $\ell \in \sigma$, and then by the definition of $\check{\sigma}$ our inequality means that the quotient of the transform of $u^{n}$ by the transform of $u^{m^{t}}$ is in $k[\check{\sigma} \cap M]$, which means that $u^{n} k[\check{\sigma} \cap M] \subset \mathscr{M} k[\check{\sigma} \cap M]$. For this to be true in all charts it is necessary and sufficient, as we saw, that $n$ should be in the convex hull of $\bigcup_{1 \leq s \leq q}\left(m^{s}+\mathbf{R}_{\geq 0}^{d}\right)$. So we have finally, using a little sheaf-theoretic language 
(in particular, $u^{n} \mathscr{O}_{Z(\Sigma)}=u^{n} \circ \pi(\Sigma)$ viewed as a global section of the sheaf $\left.\mathscr{O}_{Z(\Sigma)}\right):$

Lemma 3.2. $u^{n} \mathscr{O}_{Z(\Sigma)} \in \mathscr{M} \mathscr{O}_{Z(\Sigma)}$ if and only if $n$ is in the convex hull of $\bigcup_{1 \leq s \leq q}\left(m^{s}+\mathbf{R}_{\geq 0}^{d}\right)$.

Now one defines integral dependance over an ideal (a concept which goes back to Prüfer or even Dedekind) as follows:

Definition. An element $h$ of a commutative ring $R$ is integral over an ideal $I$ of $R$ if it satisfies an algebraic relation

$$
h^{r}+a_{1} h^{r-1}+\cdots+a_{r}=0, \quad \text { with } a_{i} \in I^{i} \text { for } 1 \leq i \leq r .
$$

It is not difficult to see that the set of elements integral over $I$ is an ideal $\bar{I}$ containing $I$ and contained in $\sqrt{I}$; it is the integral closure of $I$. We have the following characterization in algebraic geometry, which follows from the Riemann extension theorem:

Proposition 3.3 [Lipman and Teissier 1981]. Let $k$ be a field and $R$ a localization of a finitely generated reduced $k$-algebra. Let $I$ be an ideal of $R$ and $h \in R$. The element $h$ is integral over $I$ if and only if there exists a proper and birational morphism $t: Z \rightarrow \operatorname{Spec} R$ such that $h \circ t \in I \mathscr{O}_{Z}$ (i.e., $h \mathscr{O}_{Z} \in I \mathscr{O}_{Z}$ ), and then this inclusion holds for any such morphism such that $Z$ is normal and $I \mathscr{O}_{Z}$ is invertible.

From this follows the interpretation of Lemma 3.2:

Proposition 3.4. The integral closure in $k\left[u_{1}, \ldots, u_{d}\right]$ of a monomial ideal generated by the monomials $u^{m^{1}}, \ldots, u^{m^{q}}$ is the monomial ideal generated by the monomials with exponents in the convex hull $\bar{E}$ of $E=\bigcup_{1 \leq s \leq q}\left(m^{s}+\mathbf{R}_{\geq 0}^{d}\right)$.

EXAMPLE. In the ring $k\left[u_{1}, \ldots, u_{d}\right]$, for each integer $n \geq 1$ the integral closure of the ideal generated by $u_{1}^{n}, \ldots, u_{d}^{n}$ is $\left(u_{1}, \ldots, u_{d}\right)^{n}$.

EXERCISE. Check that in the preceding subsection, one can in all statements and proofs replace the positive quadrant of $\mathbf{R}^{d}$ by any strictly convex rational cone $\sigma_{0} \subset \mathbf{R}^{d}$ and let $\mathscr{M}$ denote the ideal generated by monomials $u^{m^{1}}, \ldots, u^{m^{q}}$ of the normal toric algebra $k\left[\check{\sigma}_{0} \cap M\right]$; its integral closure $\overline{\mathscr{M}}$ in that algebra is generated by the monomials with exponents in the convex hull in $\check{\sigma}_{0}$ of $\bigcup_{1 \leq s \leq q}\left(m^{s}+\check{\sigma}_{0}\right)$.

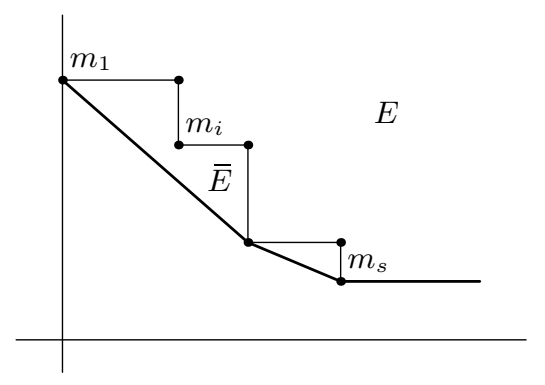




\section{The Monomial Briançon-Skoda Theorem}

Theorem 4.1 (CARAthÉOdory). Let $E$ be a subset of $\mathbf{R}^{d}$; every point of the convex hull of $E$ is in the convex hull of $d+1$ points of $E$.

Proof. For the reader's convenience, here is a sketch of the proof, according to [Grünbaum 1967]. First one checks that the convex hull of $E$, defined as the intersection of all convex subsets of $\mathbf{R}^{d}$ containing $E$, coincides with the set of points of $\mathbf{R}^{d}$ which are in the convex hull of a finite number of points of $E$ :

Given a finite set $F$ of points of $E$, its convex hull $\bar{F}$ is contained in the convex hull $\bar{E}$ of $E$. Now for two finite sets $F$ and $F^{\prime}$ we have $\bar{F} \cup \bar{F}^{\prime} \subseteq \overline{F \cup F^{\prime}}$, so that $\bigcup_{F} \bar{F}$ is convex. It contains $E$ and so has to be equal to $\bar{E}$, which proves the assertion.

Given a point $x$ of the convex hull of $E$, let $p$ be the smallest integer such that $x$ is in the convex hull of $p+1$ points of $E$, i.e., that $x=\sum_{i=0}^{p} \alpha_{i} x_{i}$, with $\alpha_{i} \geq 0, \sum_{i=0}^{p} \alpha_{i}=1$ and $x_{i} \in E$; we must prove that $p \leq d$. Assume that $p>d$. Then the points $x_{i}$ must be affinely dependent: there is a relation $\sum_{i=0}^{p} \beta_{i} x_{i}=0$ with $\beta_{i} \in \mathbf{R}$, where not all the $\beta_{i}$ are zero and $\sum_{i=0}^{p} \beta_{i}=0$. We may choose the $\beta_{i}$ so that at least one is $>0$ and renumber the points $x_{i}$ so that $\beta_{p}>0$ and for each index $i$ such that $\beta_{i}>0$ we have $\alpha_{i} / \beta_{i} \geq \alpha_{p} / \beta_{p}$. For $0 \leq i \leq p-1$ set $\gamma_{i}=\alpha_{i}-\alpha_{p} / \beta_{p} \beta_{i}$, and $\gamma_{p}=0$. Now we have

$$
\sum_{i=0}^{p-1} \gamma_{i} x_{i}=\sum_{i=0}^{p} \gamma_{i} x_{i}=\sum_{i=0}^{p} \alpha_{i} x_{i}-\frac{\alpha_{p}}{\beta_{p}} \sum_{i=0}^{p} \beta_{i} x_{i}=x,
$$

and moreover

$$
\sum_{i=0}^{p-1} \gamma_{i}=\sum_{i=0}^{p} \gamma_{i}=\sum_{i=0}^{p} \alpha_{i}-\frac{\alpha_{p}}{\beta_{p}} \sum_{i=0}^{p} \beta_{i}=1
$$

Finally, each $\gamma_{i}$ is indeed $\geq 0$ since if $\beta_{i} \leq 0$ we have $\gamma_{i} \geq \alpha_{i} \geq 0$ and if $\beta_{i}>0$ then by our choice of numbering we have $\gamma_{i}=\beta_{i}\left(\alpha_{i} / \beta_{i}-\alpha_{p} / \beta_{p}\right) \geq 0$. Assuming that $p>d$ we have expressed $x$ as the barycenter of the $p$ points $x_{0}, \ldots, x_{p-1}$ of $E$ with coefficients $\gamma_{i}$, which contradicts the definition of $p$ and thus proves the theorem.

Taking for $E$ the set consisting of $d+1$ affinely independent points of $\mathbf{R}^{d}$ shows that the bound of the theorem is optimal. However, the following result means that this is essentially the only case where $d+1$ points are necessary:

Proposition 4.2 [Fenchel 1929; Hanner and Rådström 1951]. Let $E \subset \mathbf{R}^{d}$ be a subset having at most $d$ connected components. Every point of the convex hull of $E$ is in the convex hull of d points of $E$.

Proof. We follow [Hanner and Rådström 1951]. Assume that a point $m$ of the convex hull is not in the convex hull of any subset of $d$ points of $E$. By Caratheodory's theorem, $m$ is in the convex hull $\tau \subset \mathbf{R}^{d}$ of $d+1$ points of $E$; if these $d+1$ points were not linearly independent, the point $m$ would be 
in the convex hull of the intersection of $E$ with a hyperplane and we could apply Caratheodory's theorem in a space of dimension $d-1$ and contradict our assumption, so the convex hull $\tau$ of the $d+1$ points is a $d$-simplex. Choose $m$ as origin, and denote by $\left(q_{0}, \ldots, q_{d}\right)$ the vertices of $\tau$. We have therefore $0=\sum_{0}^{d} r_{i} q_{i}$ with $r_{i} \geq 0$ and $\sum_{0}^{d} r_{i}=1$. Our assumption that 0 is not the barycenter of $d$ points implies that 0 is in the interior of $\tau$, that is, $r_{i}>0$ for $0 \leq i \leq d$. Consider the simplex $-\tau$ and the cones with vertex 0 drawn on the faces of $-\tau$. Since the $r_{i}$ are $>0$, we can reinterpret the expression of 0 as a barycenter of the $q_{i}$ to mean that each $q_{i}$ is in the cone with vertex 0 generated by the vectors $-q_{j}$ for $j \neq i$; thus each of these cones drawn from 0 on the faces of $-\tau$ contains a point of $E$, namely one of the $q_{i}$. The union of their closures is $\mathbf{R}^{d}$ because $-\tau$ is a $d$-simplex, and no point of $E$ can be on the boundary of one of these cones; if such was the case, this point, together with $d-1$ of the vertices of $\tau$, would define a $(d-1)$-simplex with vertices in $E$ and containing 0 , a possibility which we have excluded. Therefore these $d+1$ cones divide $E$ into $d+1$ disjoint nonempty parts, and $E$ must have at least $d+1$ connected components.

We remark that, given finitely many points $m^{1}, \ldots, m^{q}$ in the positive quadrant $\mathbf{R}_{\geq 0}^{d}$, the set $E=\bigcup_{s=1}^{q}\left(m^{s}+\mathbf{R}_{\geq 0}^{d}\right)$ is connected. Indeed, by definition, each point of this set is connected by a line to at least one of the points $m^{s}$, and any point of $\mathbf{R}_{\geq 0}^{d}$ having each of its coordinates larger than the maximum over $s \in\{1, \ldots, q\}$ of the corresponding coordinate of the $m^{s}$ is in $E$ and connected by lines to all the points $m^{s}$, so that any two of the points $m^{s}$ are connected in $E$.

Now let $\sigma$ be a strictly convex rational cone in $\check{\mathbf{R}}^{d}$ and $\check{\sigma} \subset \mathbf{R}^{d}$ its dual. We need not assume that $\sigma$ is regular, or even simplicial. Let $m^{1}, \ldots, m^{q}$ be integral points in $\check{\sigma}$, corresponding to monomials $u^{m^{1}}, \ldots, u^{m^{q}}$ in the algebra $k[\check{\sigma} \cap M]$. The integral closure $\overline{\mathscr{M}}$ in $k[\check{\sigma} \cap M]$ of the ideal $\mathscr{M}$ generated by the monomials $u^{m^{s}}$ is the ideal generated by the monomials $u^{n}$ such that $n$ is in the convex hull of the set $E=\bigcup_{s=1}^{q}\left(m^{s}+\check{\sigma}\right)$. What we have just said about the connectedness of $E$ extends immediately.

Theorem 4.3 (Monomial Briançon-Skoda theorem). Let $k$ be a field and let $\sigma$ be a strictly convex rational cone in $\mathbf{R}^{d}$. Given a monomial ideal $\mathscr{M}$ in $k[\check{\sigma} \cap M]$, we have the inclusion of ideals

$$
\overline{\mathscr{M}^{d}} \subset \mathscr{M} .
$$

Proof. (Compare with [Teissier 1988].) Let $y_{1}, \ldots, y_{N}$ be a system of homogeneous generators of the graded $k$-algebra $k[\check{\sigma} \cap M]$ and let $y^{m^{1}}, \ldots, y^{m^{q}}$ be generators of $\mathscr{M}$ in $k[\check{\sigma} \cap M]$. Set $E=\bigcup_{1 \leq s \leq q}\left(m^{s}+\check{\sigma}\right) \subset \check{\sigma}$. Thanks to Proposition 4.2 and the fact that $E$ is connected, it suffices to show that any point $n \in \check{\sigma} \cap M$ which is the barycenter of $d$ points $x_{1}, \ldots, x_{d}$, each of which is the 
sum of $d$ points of $E$, is in $E$. But then $n / d$ is also, as a barycenter of barycenters of points of $E$, in the convex hull of $E$, and therefore, by Proposition 4.2, the barycenter of $d$ points of $E$. Write $n / d=\sum_{i=1}^{d} r_{i} e_{i}$ with $e_{i} \in E, r_{i} \geq 0$ and $\sum_{i=1}^{d} r_{i}=1$. At least one of the $r_{i}$, say $r_{1}$, must be at least $1 / d$, so that $n \in e_{1}+\check{\sigma} \subset E$, which proves the result.

Exercise. Prove by the same method that for each integer $\lambda \geq 1$ we have

$$
\overline{\mathscr{M}^{d+\lambda-1}} \subset \mathscr{M}^{\lambda} \text {. }
$$

REMARK. It is not difficult to check that

$$
\bar{E}=\lim _{n \rightarrow \infty} \frac{n E}{n}=\bigcup_{n \in \mathbf{N}} \frac{n E}{n},
$$

where $n E$ is the Minkowski multiple (the set of all sums of $n$ elements of $E$ ) and division by $n$ means a homothety of ratio $1 / n$. In fact, the inclusion $\bigcup_{n \in \mathbf{N}}(n E / n) \subset \bar{E}$ is clear, and the first set is also clearly convex, so they are equal. The combinatorial avatar of the weak form of the Briançon-Skoda theorem, which states that $x \in \overline{\mathscr{M}}$ implies $x^{d} \in \mathscr{M}$, is the existence of a uniform bound for the $n$ such that $x \in \bar{E}$ implies $n x \in E$, namely $n=d$.

The Briançon-Skoda theorem is the statement $\overline{\mathscr{M}^{d}} \subset \mathscr{M}$ for an ideal in a $d$ dimensional regular local ring. The rings $k[\check{\sigma} \cap M]$ are not regular in general, nor are they local, but the monomial Briançon-Skoda theorem for ideals in their localizations $k[\check{\sigma} \cap M]_{m}$ follows from the results of [Lipman and Teissier 1981] in the case where $\mathscr{M}$ contains an ideal generated by a regular sequence and with the same integral closure, since $k[\check{\sigma} \cap M]$ has only rational singularities (see [Kempf et al. 1973]) and hence $k[\check{\sigma} \cap M]_{m}$ is a pseudorational local ring.

The Briançon-Skoda theorem was originally proved [Skoda and Briançon 1974] by analytic methods for ideals of $\mathbf{C}\left\{z_{1}, \ldots, z_{d}\right\}$, and has been the subject of many algebraic proofs and generalizations. The first algebraic proof was given in [Lipman and Teissier 1981], albeit for a restricted class of ideals in an extended class of rings (pseudorational ones). See [Hochster 2004] and [Blickle and Lazarsfeld 2004] in this volume for references and recent developments.

\section{Polynomial Ideals and Nondegeneracy}

The hypothesis of nondegeneracy of a polynomial with respect to its Newton polyhedron has a fairly ancient history in the sense that it was made more or less implicitely by authors trying to compute various invariants of a projective hypersurface from its Newton polyhedron. In the nineteenth century one may mention Minding and Elliott, and in the twentieth Baker (1905) and Hodge (1930). The last three were interested in computing the geometric genus of a projective curve or surface with isolated singularities from its Newton polygon or polyhedron. This is a special case of computation of a multiplier ideal. See 
[Merle and Teissier 1980], and compare its Theorem of Hodge 2.3.1 with the recent work of J. Howald expounded in [Blickle and Lazarsfeld 2004]; see also [Howald 2001].

The modern approach to nondegeneracy was initiated essentially by Kushnirenko [1976] and Khovanskii, who made the nondegeneracy condition explicit and computed from the Newton polyhedron invariants of a similar nature. In particular Khovanskii gave the general form of Hodge's result. The essential facts behind the classical computations turned out to be that nondegenerate singularities have embedded toric (pseudo-)resolutions which depend only on their Newton polyhedron and from which one can read combinatorially various interesting invariants, and that in the spaces of coefficients of all those functions or systems of functions having given polyhedra, those which are nondegenerate are Zariski-dense.

Let $f=\sum_{p} f_{p} u^{p}$ be an arbitrary polynomial or power series in $d$ variables with coefficients in the field $k$. Let $\operatorname{Supp} f=\left\{p \in \mathbf{R}^{d}: f_{p} \neq 0\right\}$ be its support. The affine Newton polyhedron of $f$ in the coordinates $\left(u_{1}, \ldots, u_{d}\right)$ is the boundary $\mathscr{N}(f)$ of the convex hull in $\mathbf{R}_{\geq 0}^{d}$ of the support of $f$. The local Newton polyhedron is the boundary $\mathscr{N}_{+}(f)$ of

$$
\mathscr{P}_{+}(f)=\text { convex hull of }\left(\operatorname{Supp} f+\mathbf{R}_{\geq 0}^{d}\right) .
$$

It has finitely many compact faces and its noncompact faces of dimension at most $d-1$ are parallel to coordinate hyperplanes. Both polyhedra depend not only on $f$ but also on the choice of coordinates. Remark also that the local Newton polyhedron is of no interest if $f$ has a nonzero constant term.

We can define the affine and the local support functions associated with the function $f$ as follows (in the affine case, this is the same definition as before, applied to the set of monomials appearing in $f$ ):

For the affine Newton polyhedron it is the function defined on $\check{\mathbf{R}}^{d}$ by

$$
\mathrm{h}_{\mathscr{N}(f)}(\ell)=\min _{p \in \mathscr{N}(f)} \ell(p),
$$

and for the local Newton polyhedron it is defined on the first quadrant $\check{\mathbf{R}}_{\geq 0}^{d}$ by

$$
\mathrm{h}_{\mathscr{N}_{+}(f)}(\ell)=\min _{p \in \mathscr{N}_{+}(f)} \ell(p) .
$$

Both functions are piecewise linear in their domain of definition, meaning that there is a decomposition of the domain of definition into convex cones such that the function is linear in each cone. These collections of cones are actually fans, in $\check{\mathbf{R}}^{d}$ and $\check{\mathbf{R}}_{\geq 0}^{d}$ respectively. These fans are "dual" to the Newton polyhedra in the following sense:

Consider, say for the local polyhedron, the following equivalence relation between linear functions:

$\ell \equiv \ell^{\prime} \Longleftrightarrow\left\{p \in \mathscr{N}_{+}(f): \ell(p)=\mathrm{h}_{\mathscr{N}_{+}(f)}(\ell)\right\}=\left\{p \in \mathscr{N}_{+}(f): \ell^{\prime}(p)=\mathrm{h}_{\mathscr{N}_{+}(f)}\left(\ell^{\prime}\right)\right\}$. 
Its equivalence classes form a decomposition of the first quadrant into strictly convex rational cones, and by definition the support function is linear in each of them, given by $\ell \mapsto \ell(p)$ for any $p$ in the set $\left\{p \in \mathscr{N}_{+}(f): \ell(p)=\mathrm{h}_{\mathscr{N}_{+}(f)}(\ell)\right\}$. These sets are faces of the Newton polyhedron, and the collection of the cones constitutes a fan $\Sigma_{\mathscr{N}}$ in $\mathbf{R}_{\geq 0}^{d}$, called the dual fan of the Newton polyhedron. This establishes a one-to-one decreasing correspondence between the cones of the dual fan of a Newton polyhedron and the faces of all dimensions of that Newton polyhedron. Corresponding to noncompact faces of the Newton polyhedron meet coordinate hyperplanes outside the origin.

We have now associated to each polynomial $f=\sum_{p} f_{p} u^{p}$ a dual fan in $\check{\mathbf{R}}^{d}$ corresponding to the global Newton polyhedron, and another in $\check{\mathbf{R}}_{\geq 0}^{d}$ corresponding to the local Newton polyhedron. The local polyhedron is also defined for a series $f=\sum_{p} f_{p} u^{p}$, and the combinatorial constructions are the same. For the moment, let's restrict our attention to the local polyhedron, assuming that $f_{0}=0$, and let's choose a regular refinement $\Sigma$ of the fan associated to it.

By the definition just given, this means that for each cone $\sigma=\left\langle a^{1}, \ldots, a^{k}\right\rangle$ of the fan $\Sigma$, the primitive vectors $a^{i}$ form part of a basis of the integral lattice, and all the linear forms $p \mapsto\left\langle a^{i}, p\right\rangle$, when restricted to the set $\left\{p: f_{p} \neq 0\right\}$, take their minimum value on the same subset, which is a face, of the (local) Newton polyhedron of $f=\sum_{p} f_{p} u^{p}$. This face may or may not be compact.

We examine the behavior of $f$ under the map $\pi(\sigma): Z(\sigma) \rightarrow \mathbf{A}^{d}(k)$ corresponding to a cone $\sigma=\left\langle a^{1}, \ldots, a^{d}\right\rangle \subset \check{\mathbf{R}}_{\geq 0}^{d}$ of a regular fan which is a subdivision of the fan associated to the local polyhedron of $f$. If we write $\mathrm{h}$ for $\mathrm{h}_{\mathscr{N}_{+}(f)}$ we get

$$
\begin{aligned}
f \circ \pi(\sigma) & =\sum_{p} f_{p} y_{1}^{\left\langle a^{1}, p\right\rangle} \ldots y_{d}^{\left\langle a^{d}, p\right\rangle} \\
& =y_{1}^{h\left(a^{1}\right)} \ldots y_{d}^{h\left(a^{d}\right)} \sum_{p} f_{p} y_{1}^{\left\langle a^{1}, p\right\rangle-h\left(a^{1}\right)} \ldots y_{d}^{\left\langle a^{d}, p\right\rangle-h\left(a^{d}\right)} .
\end{aligned}
$$

The last sum is by definition the strict transform of $f$ by $\pi(\sigma)$.

EXERCISES. Check that:

(a) In each chart $Z(\sigma)$ the exceptional divisor consists (set-theoretically) of the union of those hyperplanes $y_{j}=0$ such that $a^{j}$ is not a basis vector of $\check{\mathbf{Z}}^{d}$.

(b) Provided that no monomial in the $u_{i}$ divides $f$, the hypersurface

$$
\sum_{p} f_{p} y_{1}^{\left\langle a^{1}, p\right\rangle-h\left(a^{1}\right)} \ldots y_{d}^{\left\langle a^{d}, p\right\rangle-h\left(a^{d}\right)}=0
$$

is indeed the strict transform by the map $\pi(\sigma): Z(\sigma) \rightarrow \mathbf{A}^{d}(k)$ of the hypersurface $X \subset \mathbf{A}^{d}(k)$ defined by $f\left(u_{1}, \ldots, u_{d}\right)=0$, in the sense that it is the closure in $Z(\sigma)$ of the image of $X \cap\left(k^{*}\right)^{d}$ by the isomorphism induced by $\pi(\sigma)$ on the tori of the two toric varieties $Z(\sigma)$ and $\mathbf{A}^{d}(k)$ as well as in the sense 
that it is obtained from $f \circ \pi(\sigma)$ by factoring out as many times as possible the defining functions of the components of the exceptional divisor.

Denote by $\tilde{f}$ the strict transform of $f$ and note that by construction it has a nonzero constant term: the cone $\sigma$ is of maximal dimension, which means that there is a unique exponent $p$ such that $\langle a, p\rangle=h(a)$ for $a \in \sigma$.

The map $\pi(\tau)$ associated to a face $\tau$ of $\sigma$ coincides with the restriction of $\pi(\sigma)$ to an open set $Z(\tau) \subset Z(\sigma)$ which is of the form $y_{j} \neq 0$ for $j \in J$, where $J$ depends on $\tau \subset \sigma$.

Now we can, for each cone $\sigma$ of our regular fan, stratify the space $Z(\sigma)$ in such a way that $\pi(\sigma)^{-1}(0)$ is a union of strata. Let $I$ be a subset of $\{1,2, \ldots, d\}$ and define $S_{I}$ to be the constructible subset of $Z(\sigma)$ defined by $y_{i}=0$ for $i \in I, y_{i} \neq 0$ for $i \notin I$. The $S_{I}$ for $I \subset\{1,2, \ldots, d\}$ constitute a partition of $Z(\sigma)$ into nonsingular varieties, constructible in $Z(\sigma)$, which we call the natural stratification of $Z(\sigma)$. If we glue up two charts $Z(\sigma)$ and $Z\left(\sigma^{\prime}\right)$ along $Z\left(\sigma \cap \sigma^{\prime}\right)$, the natural stratifications glue up as well.

If we restrict the strict transform

$$
\tilde{f}\left(y_{1}, \ldots, y_{d}\right)=\sum_{p} f_{p} y_{1}^{\left\langle a^{1}, p\right\rangle-h\left(a^{1}\right)} \ldots y_{d}^{\left\langle a^{d}, p\right\rangle-h\left(a^{d}\right)}
$$

to a stratum $S_{I}$, we see that in the sum representing $\tilde{f}\left(y_{1}, \ldots, y_{d}\right)$ only the terms $f_{p} y_{1}^{\left\langle a^{1}, p\right\rangle-h\left(a^{1}\right)} \ldots y_{d}^{\left\langle a^{d}, p\right\rangle-h\left(a^{d}\right)}$ such that $\left\langle a^{i}, p\right\rangle-h\left(a^{i}\right)=0$ for $i \in I$ survive. These equalities define a unique face $\gamma_{I}$ of the Newton polyhedron of $f$, since our fan is a subdivision of its dual fan. Given a series $f=\sum_{p} f_{p} u^{p}$ and a weight vector $\check{a} \in \mathbf{R}_{\geq 0}^{d}$, the set

$$
\left\{p \in \mathbf{Z}_{\geq 0}^{d}: f_{p} \neq 0 \text { and }\langle\check{a}, p\rangle=h(\check{a})\right\}
$$

is a face of the local Newton polyhedron of $f$, corresponding to the cone of the dual fan which contains $\check{a}$ in its relative interior. If all the coordinates of the vector $\check{a}$ are positive, this face is compact.

Moreover, if we define

$$
f_{\gamma_{I}}=\sum_{p \in \gamma_{I}} f_{p} u^{p}
$$

to be the partial polynomial associated to the face $\gamma_{I}$, which is nothing but the sum of the terms of $f$ whose exponent is in the face $\gamma_{I}$, we see that we have the fundamental equality

$$
\left.\tilde{f}\right|_{S_{I}}=\left.\widetilde{f_{\gamma_{I}}}\right|_{S_{I}}
$$

and we remark moreover that $\widetilde{f_{\gamma_{I}}}$ is a function on $Z(\sigma)$ which is independent of the coordinates $y_{i}$ for $i \in I$, so that it is determined by its restriction to $S_{I}$.

Now, to say that the strict transform $\tilde{f}=0$ in $Z(\sigma)$ of the hypersurface $f=0$ is transversal to the stratum $S_{I}$ and is nonsingular in a neighborhood of its intersection with it is equivalent to saying that the restriction $\left.\tilde{f}\right|_{S_{I}}$ of the function $\tilde{f}$ defines, by the equation $\left.\tilde{f}\right|_{S_{I}}=0$, a nonsingular hypersurface of $S_{I}$. 
By the definition of $S_{I}$ and what we have just seen, this in turn is equivalent to saying that the equation $\widetilde{f_{\gamma_{I}}}=0$ defines a nonsingular hypersurface in the torus $\left(k^{*}\right)^{d}=\left\{u: \prod_{1}^{d} u_{j} \neq 0\right\}$ of $Z(\sigma)$, and this finally is equivalent to saying that $f_{\gamma_{I}}=0$ defines a nonsingular hypersurface in the torus $\left(k^{*}\right)^{d}$ of the affine space $\mathbf{A}^{d}(k)$ since $\pi(\sigma)$ induces an isomorphism of the two tori.

This motivates the definition:

Definition. The series $f=\sum_{p} f_{p} u^{p}$ is nondegenerate with respect to its Newton polyhedron in the coordinates $\left(u_{1}, \ldots, u_{d}\right)$ if for every compact face $\gamma$ of $\mathscr{N}_{+}(f)$ the polynomial $f_{\gamma}$ defines a nonsingular hypersurface of the torus $\left(k^{*}\right)^{d}$.

REMARK. By definition of the faces of the Newton polyhedron and of the dual fan, in each chart $Z(\sigma)$ of a regular fan refining the dual fan of $\mathscr{N}_{+}(f)$, the compact faces $\gamma_{I}$ correspond to strata $S_{I}$ of the canonical stratification which are contained in $\pi(\sigma)^{-1}(0)$. Each stratum $S_{I}$ which is not contained in $\pi(\sigma)^{-1}(0)$ contains in its closure strata which are.

Proposition 5.1. If the germ of hypersurface $X$ is defined by the vanishing of a series $f$ which is nondegenerate, there is a neighborhood $U$ of 0 in $\mathbf{A}^{d}(k)$ (a formal neighborhood if the series $f$ does not converge) such that the strict transform of $X \cap U$ by the toric map

$$
\pi(\Sigma): Z(\Sigma) \rightarrow \mathbf{A}^{d}(k)
$$

associated to a regular fan refining the dual fan of its Newton polyhedron is nonsingular and transversal in each chart to the strata of the canonical stratification.

Proof. By the fundamental equality seen above, the restriction of the strict transform to one of the strata contained in $\pi(\sigma)^{-1}(0)$, say $S_{I}$, has the same behavior as the restriction of $f_{\gamma_{I}}$, where $\gamma_{I}$ is a compact face of the Newton polyhedron of $f$, to the torus $\left(k^{*}\right)^{d}$. As we saw, this implies that the strict transform of $X \cap U$ is nonsingular and transversal to $S_{I}$. By openness of transversality the same transversality holds, whithin a neighborhood of each point of $\pi(\Sigma)^{-1}(0)$, for all strata.

Since the map $\pi(\Sigma): Z(\Sigma) \rightarrow \mathbf{A}^{d}(k)$ is proper, there is a neighborhood $U$ of 0 in $\mathbf{A}^{d}(k)$ such that the strict transform by $\pi(\Sigma)$ of the hypersurface $X \subset \mathbf{A}^{d}(k)$ is nonsingular in $\pi(\Sigma)^{-1}(U)$ and transversal in each chart $Z(\sigma)$ to all the strata of the canonical stratification.

The definition and properties of nondegeneracy extend to systems of functions as follows. Let $f_{1}, \ldots, f_{k}$ be series in the variables $u_{1}, \ldots, u_{d}$ defining a subspace $X \subset \mathbf{A}^{d}(k)$ in a neighborhood of 0 . For each $j=1, \ldots, k$ we have a local Newton polyhedron $\mathscr{N}_{+}\left(f_{j}\right)$. Choose a regular fan $\Sigma$ of $\mathbf{R}_{\geq 0}^{d}$ compatible with all the fans dual to the polyhedra $\mathscr{N}_{+}\left(f_{j}\right)$ for $j=1, \ldots, k$. We have for each $j$ the same correspondence as above between the strata $S_{I}$ of each chart $Z(\sigma)$ for $\sigma \in \Sigma$ and the faces of $\mathscr{N}_{+}\left(f_{j}\right)$, the strata contained in $\pi(\sigma)^{-1}(0)$ corresponding to compact faces. 
For each vector $\check{a} \in \check{\mathbf{R}}_{\geq 0}^{d}$ we get as above a system of equations $f_{1, \check{a}}, \ldots, f_{k, \check{a}}$, where

$$
f_{j, \check{a}}=\sum_{\{p:\langle\check{a}, p\rangle=h(\check{a})\}} f_{j p} u^{p} .
$$

Definition. The system of equations $f_{1}, \ldots, f_{k}$ is said to be nondegenerate of rank $c$ with respect to the Newton polyhedra of the $f_{j}$ in the coordinates $\left(u_{1}, \ldots, u_{d}\right)$ if for each vector $\check{a} \in \mathbf{R}_{>0}^{d}$ the ideal of $k\left[u_{1}^{ \pm 1}, \ldots, u_{d}^{ \pm 1}\right]$ generated by the polynomials $f_{1, \check{a}}, \ldots, f_{k, \check{a}}$ defines a nonsingular subvariety of dimension $d-c$ of the torus $\left(k^{*}\right)^{d}$.

EXERCISE. Check that, since we took $\check{a} \in \mathbf{R}_{>0}^{d}$ in the definition, it is equivalent to say that for each choice of a compact face $\gamma_{j}$ in each $\mathscr{N}_{+}\left(f_{j}\right)$, the ideal generated by the polynomials $f_{1 \gamma_{1}}, \ldots, f_{k \gamma_{k}}$ defines a nonsingular subvariety of dimension $d-c$ of the torus $\left(k^{*}\right)^{d}$.

Exactly as in the case of hypersurfaces, one then has:

PROPOSITION 5.2. If the system of equations $f_{1}, \ldots, f_{k}$ is nondegenerate of rank $c$, for any regular fan $\Sigma$ of $\mathbf{R}_{\geq 0}^{d}$ compatible with the dual fans of the polyhedra $\mathscr{N}_{+}\left(f_{j}\right)$, there is a neighborhood $U$ of 0 in $\mathbf{A}^{d}(k)$ (a formal neighborhood if all the series $f_{j}$ do not converge) such that the strict transform $X^{\prime} \subset Z(\Sigma)$ by the toric map $\pi(\Sigma): Z(\Sigma) \rightarrow \mathbf{A}^{d}(k)$ of the subvariety $X \cap U$ defined in $U$ by the ideal generated by $f_{1}, \ldots, f_{k}$ is nonsingular and of dimension $d-c$ and transversal to the strata of the natural stratification in $\pi(\Sigma)^{-1}(U)$.

Proof. The same as that of Proposition 5.1.

There is a difference, however, between the birational map $X^{\prime} \rightarrow X \cap U$ induced by $\pi(\Sigma)$ and a resolution of singularities; this map is not necessarily an isomorphism outside of the singular locus; it is therefore only a pseudoresolution in the sense of [Goldin and Teissier 2000]. In fact, even in the nondegenerate case, and even for a hypersurface, the Newton polyhedron contains in general far too little information about the singular locus of $X$ near 0 . Kushnirenko introduced, for isolated hypersurface singularities, the notion of being convenient with respect to a coordinate system. It means that the Newton polyhedron meets all the coordinate axis of $\mathbf{R}_{\geq 0}^{d}$. For a hypersurface with isolated singularity, it implies that a toric pseudoresolution associated to the Newton polyhedron is a resolution. This was extended and generalized by M. Oka for complete intersections. The reader is referred to [Oka 1997, Ch. III] (especially Theorem 3.4) and we will only quote here the following fact, which is also a consequence of the existence of a toric pseudoresolution:

ThEOREM [Oka 1997, Ch. III, Lemma 2.2]. If $k$ is a field and $(X, 0) \subset \mathbf{A}^{d}(k)$ is a germ of a complete intersection with equations $f_{1}=\cdots=f_{c}=0$, which is nondegenerate with respect to the Newton polyhedra of its equations in the coordinates $u_{1}, \ldots, u_{d}$, then there is a (possibly formal) neighborhood $U$ of $0 \in$ 
$\mathbf{A}^{d}(k)$ such that the intersection of $X$ and the torus $\left(k^{*}\right)^{d}$ has no singularities in $U$.

In the formal case this should be understood as saying that formal germ at 0 of the singular locus of $X$ is contained in the union of the coordinate hyperplanes.

Finally, it seems that the following coordinate-free definition of nondegeneracy is appropriate:

Definition. An algebraic or formal subscheme $X$ of an affine space $\mathbf{A}^{d}(k)$ is nondegenerate at a point $x \in X$ if there exist local coordinates $u_{1}, \ldots, u_{d}$ centered at $x$ and an open (étale or formal) neighborhood $U$ of $x$ in $\mathbf{A}^{d}(k)$ such that there is a proper birational toric map $\pi: Z \rightarrow U$ in the coordinates $u_{1}, \ldots, u_{d}$ with $Z$ nonsingular and such that the strict transform $X^{\prime}$ of $X \cap U$ by $\pi$ is nonsingular and transversal to the exceptional divisor at every point of $\pi^{-1}(x) \cap X^{\prime}$.

If $X$ admits a system of equations which in some coordinates is nondegenerate with respect to its Newton polyhedra, it is also nondegenerate in this sense as we saw. The converse will not be discussed here.

Question [Teissier 2003]. Given a reduced and equidimensional algebraic or formal space $X$ over an algebraically closed field $k$, is it true that for every point $x \in X$ there is a local formal embedding of $X$ into an affine space $\mathbf{A}^{N}(k)$ such that $X$ is nondegenerate in $\mathbf{A}^{N}(k)$ at the point $x$ ?

A subsequent problem is to give a geometric interpretation of the systems of coordinates in which an embedded toric resolution for $X$ exists.

For branches (analytically irreducible curve singularities), the question is answered positively, and the problem settled in Section 7 below. Recent work of P. González Pérez [2003] also settles question and problem for irreducible quasiordinary hypersurface singularities.

In [Teissier 2003] one finds an approach to the simpler problem where the nondegeneracy is requested only with respect to a valuation of the local ring of $X$ at $x$.

In a given coordinate system, and for given Newton polyhedra, "almost all" systems of polynomials having these given Newton polyhedra are nondegenerate with respect to them. In this sense there are many nondegenerate singularities. However, nondegenerate singularities are very special from the viewpoint of the classification of singularities. A plane complex branch is nondegenerate in some coordinate system if and only if it has only one characteristic pair, which means that its equation can be written in some coordinate system as

$$
u_{1}^{p}-u_{0}^{q}+\sum_{i / q+j / p>1} a_{i j} u_{0}^{i} u_{1}^{j}=0
$$


where $a_{i j} \in k$ and the integers $p, q$ are coprime. The curve

$$
\left(u_{1}^{2}-u_{0}^{3}\right)^{2}-u_{0}^{5} u_{1}=0
$$

is degenerate in any coordinate system since it has two characteristic pairs [Smith 1873; Brieskorn and Knörrer 1986].

\section{Resolution of Binomial Varieties}

This section presents what is in a way the simplest class of nondegenerate singularities, according to the results in [González Pérez and Teissier 2002]:

Let $k$ be a field. Binomial varieties over $k$ are irreducible varieties of the affine space $\mathbf{A}^{d}(k)$ which can, in a suitable coordinate system, be defined by the vanishing of binomials in these coordinates, which is to say expressions of the form $u^{m}-\lambda_{m n} u^{n}$ with $\lambda_{m n} \in k^{*}$. An ideal generated by such binomial expressions is called a binomial ideal. These affine varieties defined by prime binomial ideals are also the irreducible affine varieties on which a torus of the same dimension acts algebraically with a dense orbit (see [Sturmfels 1996]); they are the (not necessarily normal) affine toric varieties.

Binomial ideals were studied in [Eisenbud and Sturmfels 1996]; these authors showed in particular that if $k$ is algebraically closed their geometry is determined by the lattice generated by the differences $m-n$ of the exponents of the generating binomials. If the field $k$ is not algebraically closed, the study becomes more complicated. Here I will assume throughout that $k$ is algebraically closed. It is natural to study the behavior of binomial ideals under toric maps.

Let $\sigma=\left\langle a^{1}, \ldots, a^{d}\right\rangle$ be a regular cone in $\check{\mathbf{R}}_{\geq 0}^{d}$. The image of a binomial

$$
u^{m}-\lambda_{m n} u^{n}
$$

under the map $k\left[u_{1}, \ldots, u_{d}\right] \rightarrow k\left[y_{1}, \ldots, y_{d}\right]$ determined by $u_{i} \mapsto y_{1}^{a_{i}^{1}} \ldots y_{d}^{a_{i}^{d}}$ is given by

$$
u^{m}-\lambda_{m n} u^{n} \mapsto y_{1}^{\left\langle a^{1}, m\right\rangle} \ldots y_{d}^{\left\langle a^{d}, m\right\rangle}-\lambda_{m n} y_{1}^{\left\langle a^{1}, n\right\rangle} \ldots y_{d}^{\left\langle a^{d}, n\right\rangle} .
$$

In general this only tells us that the transform of a binomial is a binomial, which is no news since by definition of a toric map the transform of a monomial is a monomial.

However, something interesting happens if we assume that the cone $\sigma$ is compatible with the hyperplane $H_{m-n}$ which is the dual in the space of weights of the vector $m-n$ of the space of exponents, in the sense of definition on page 215, where we remember that the origin $\{0\}$ is a face of any polyhedral cone. Note that the Newton polyhedron of a binomial has only one compact face, which is a segment, so that for a cone in $\check{\mathbf{R}}_{\geq 0}^{d}$, being compatible with the hyperplane $H_{m-n}$ is the same as being compatible with the dual fan of the Newton polyhedron of our binomial. 
Let us assume that the binomial hypersurface $u^{m}-\lambda_{m n} u^{n}=0$ is irreducible; this means that no variable $u_{j}$ appears in both monomials, and the vector $m-n$ is primitive. In the sequel, I will tacitly assume this and also that our binomial is really singular, that is, not of the form $u_{1}-\lambda u^{m}$.

If the convex cone $\sigma$ of dimension $d$ is compatible with the hyperplane $H_{m-n}$, it is contained in one of the closed half-spaces determined by $H_{m-n}$. This means that all the nonzero $\left\langle a^{i}, m-n\right\rangle$ have the same sign, say positive. It also means that, if we renumber the vectors $a^{i}$ in such a way that $\left\langle a^{i}, m-n\right\rangle=0$ for $1 \leq i \leq t$ and $\left\langle a^{i}, m-n\right\rangle>0$ for $t+1 \leq i \leq d$, we can write the transform of our binomial as

$$
u^{m}-\lambda_{m n} u^{n} \mapsto y_{1}^{\left\langle a^{1}, n\right\rangle} \ldots y_{d}^{\left\langle a^{d}, n\right\rangle}\left(y_{t+1}^{\left\langle a^{t+1}, m-n\right\rangle} \ldots y_{d}^{\left\langle a^{d}, m-n\right\rangle}-\lambda_{m n}\right)
$$

And we can see that the strict transform $y_{t+1}^{\left\langle a^{t+1}, m-n\right\rangle} \ldots y_{d}^{\left\langle a^{d}, m-n\right\rangle}-\lambda_{m n}=0$ of our hypersurface in the chart $Z(\sigma)$ is nonsingular!

It is also irreducible in view of the results of [Eisenbud and Sturmfels 1996] because we assumed that the vector $m-n$ is primitive and the matrix $\left(a_{j}^{i}\right)$ is unimodular. This implies that the vector $\left(0, \ldots, 0,\left\langle a^{t+1}, m-n\right\rangle, \ldots,\left\langle a^{d}, m-n\right\rangle\right)$ is also primitive, and the strict transform irreducible. Moreover, in the chart $Z(\sigma)$ with $\sigma=\left\langle a^{1}, \ldots, a^{d}\right\rangle$, the strict transform meets the hyperplane $y_{j}=0$ if and only if $\left\langle a^{j}, m-n\right\rangle=0$. Unless our binomial is nonsingular, a case we excluded, this implies that $a^{j}$ is not a vector of the canonical basis of $W$, so that $y_{j}=0$ is a component of the exceptional divisor. So we see that the strict transform meets the exceptional divisor only in those charts such that $\sigma \cap H_{m-n} \neq\{0\}$, and then meets it transversally.

So we have in this very special case achieved that the total transform of our irreducible binomial hypersurface defines in each chart a divisor with normal crossings that is, a divisor locally at every point defined in suitable local coordinates by the vanishing of a monomial and whose irreducible components are nonsingular.

Now we consider a prime binomial ideal of $k\left[u_{1}, \ldots, u_{d}\right]$ generated by $\left(u^{m^{\ell}}-\right.$ $\left.\lambda_{\ell} u^{n^{\ell}}\right)_{\ell \in\{1, \ldots, L\}}, \lambda_{\ell} \in k^{*}$. Let us denote by $\mathscr{L}$ the sublattice of $\mathbf{Z}^{d}$ generated by the differences $m^{\ell}-n^{\ell}$. According to [Eisenbud and Sturmfels 1996], the dimension of the subvariety $X \subset \mathbf{A}^{d}(k)$ defined by the ideal is $d-r$ where $r$ is the rank of the $\mathbf{Q}$-vector space $\mathscr{L} \otimes_{\mathbf{Z}} \mathbf{Q}$. To each binomial is associated a hyperplane $H_{\ell} \subset \check{\mathbf{R}}^{d}$, the dual of the vector $m^{\ell}-n^{\ell} \in \mathbf{R}^{d}$. The intersection $\mathscr{W}$ of the hyperplanes $H_{\ell}$ is the dual of the vector subspace $\mathscr{L} \otimes \mathbf{z} \mathbf{R}$ of $\mathbf{R}^{d}$ generated by the vectors $m^{\ell}-n^{\ell}$; its dimension is $d-r$.

Let $\Sigma$ be a fan with support $\mathbf{R}_{\geq 0}^{d}$ which is compatible with each of the hyperplanes $H_{\ell}$. Let us compute the transforms of the generators $u^{m^{\ell}}-\lambda_{\ell} u^{n^{\ell}}$ in a chart $Z(\sigma)$ associated to the cone $\sigma=\left\langle a^{1}, \ldots, a^{d}\right\rangle$ : after renumbering the vectors $a^{j}$ and possibly exchanging some $m^{\ell}, n^{\ell}$ and replacing $\lambda_{\ell}$ by its inverse, we may assume that $a^{1}, \ldots, a^{t}$ are in $\mathscr{W}$, that the $\left\langle a^{j}, m^{\ell}-n^{\ell}\right\rangle$ are $\geq 0$ for 
$j=t+1, \ldots, d$, and that moreover for each such index $j$ there is an $\ell$ such $\left\langle a^{j}, m^{\ell}-n^{\ell}\right\rangle>0$. The transforms of the binomials can be written

$$
y_{1}^{\left\langle a^{1}, n^{\ell}\right\rangle} \ldots y_{d}^{\left\langle a^{d}, n^{\ell}\right\rangle}\left(y_{t+1}^{\left\langle a^{t+1}, m^{\ell}-n^{\ell}\right\rangle} \ldots y_{d}^{\left\langle a^{d}, m^{\ell}-n^{\ell}\right\rangle}-\lambda_{\ell}\right)
$$

with that additional condition. If $\sigma \cap \mathscr{W}=\{0\}$, we have $t=0$ and the subvariety defined by the equations just written (the strict transform of $X$ in $Z(\sigma)$ ) does not meet any coordinate hyperplane; in particular it does not meet the exceptional divisor. In general, still assuming that none of the binomials is already in the form $u_{j}-\lambda u^{m}$, one sees that the additional condition implies that, just like in the case of hypersurfaces, the strict transform meets the hyperplane $y_{j}=0$ if and only if $a^{j}$ is in $\mathscr{W}$.

Now the claim is that in each chart $Z(\sigma)$ the strict transform is either empty or nonsingular and transversal to the exceptional divisor.

The $\mathbf{Q}$-vector space generated by the $m^{\ell}-n^{\ell}$ is of dimension $r$. Let us assume that $m^{1}-n^{1}, \ldots, m^{r}-n^{r}$ generate it and let us denote by $\mathscr{L}_{1}$ the lattice which they generate in $\mathbf{Z}^{d}$. By construction, the quotient $\mathscr{L} / \mathscr{L}_{1}$ is a torsion $\mathbf{Z}$-module. Let us first show that the strict transform of the subspace $X_{1} \subset X$ defined by the first $r$ binomial equations is nonsingular and transversal to the exceptional divisor.

We consider then, for each cone $\sigma=\left\langle a^{1}, \ldots, a^{d}\right\rangle$, the equations

$$
\begin{aligned}
& y_{1}^{\left\langle a^{1}, m^{1}-n^{1}\right\rangle} \cdots y_{d}^{\left\langle a^{d}, m^{1}-n^{1}\right\rangle}-\lambda_{1}=0 \\
& y_{1}^{\left\langle a^{1}, m^{2}-n^{2}\right\rangle} \ldots y_{d}^{\left\langle a^{d}, m^{2}-n^{2}\right\rangle}-\lambda_{2}=0 \\
& \text {.............................. } \\
& y_{1}^{\left\langle a^{1}, m^{r}-n^{r}\right\rangle} \cdots y_{d}^{\left\langle a^{d}, m^{r}-n^{r}\right\rangle}-\lambda_{r}=0
\end{aligned}
$$

of the strict transform of $X_{1}$ in $Z(\sigma)$.

We can compute by logarithmic differentiation their jacobian matrix $J$, and find with the same definition of $t$ as above an equality of $d \times r$ matrices:

$$
y_{t+1} \ldots y_{d} J=y_{t+1}^{\sum_{s}\left\langle a^{t+1}, m^{s}-n^{s}\right\rangle} \ldots y_{d}^{\sum_{s}\left\langle a^{d}, m^{s}-n^{s}\right\rangle}\left(\left\langle a^{j}, m^{s}-n^{s}\right\rangle\right)_{1 \leq j \leq d, 1 \leq s \leq r} .
$$

Lemma 6.1. Given an irreducible binomial variety $X \subset \mathbf{A}^{d}(k)$, with the notations just introduced, for any regular cone $\sigma=\left\langle a^{1}, \ldots, a^{d}\right\rangle$ compatible with the hyperplanes $H_{\ell}$, the image in $\operatorname{Mat}_{d \times L}(k)$ of the matrix

$$
\left(\left\langle a^{j}, m^{s}-n^{s}\right\rangle\right)_{1 \leq j \leq d, 1 \leq s \leq L} \in \operatorname{Mat}_{d \times L}(\mathbf{Z})
$$

has rank $r$.

Proof. Since the vectors $a^{j}$ form a basis of $\mathbf{Q}^{d}$, and the space $\check{\mathscr{W}}=\mathscr{L} \otimes \mathbf{z} \mathbf{R}$ generated by the $m^{s}-n^{s}$ is of dimension $r$, the rank of the matrix $\left(\left\langle a^{j}, m^{s}-n^{s}\right\rangle\right)$ is $r$, which proves the lemma if $k$ is of characteristic zero. If the field $k$ is of positive characteristic the proof is a little less direct; see [Teissier 2003, Ch. 6]. 
In particular, the rank of the image in $\operatorname{Mat}_{d \times r}(k)$ of the matrix $\left(\left\langle a^{j}, m^{s}-\right.\right.$ $\left.\left.n^{s}\right\rangle\right)_{1 \leq j \leq d, 1 \leq s \leq r} \in \operatorname{Mat}_{d \times r}(\mathbf{Z})$ is $r$.

LEMma 6.2. The strict transform $X_{1}^{\prime}$ by $\pi(\Sigma)$ of the subspace $X \subset \mathbf{A}^{d}(k)$ defined by the ideal of $k\left[u_{1}, \ldots, u_{d}\right]$ generated by the binomials

$$
u^{m^{1}}-\lambda_{1} u^{n^{1}}, \ldots, u^{m^{r}}-\lambda_{r} u^{n^{r}}
$$

is regular and transversal to the exceptional divisor.

Proof. Let $\sigma$ be a cone of of maximal dimension in the fan $\Sigma$. In the chart $Z(\sigma)$, none of the coordinates $y_{t+1}, \ldots, y_{d}$ vanishes on the strict transform $X_{1}^{\prime}$ of $X_{1}$ and the equations of $X_{1}^{\prime}$ in $Z(\sigma)$ are independent of $y_{1}, \ldots, y_{t}$. Therefore to prove that the jacobian $J$ of the equations has rank $r$ at each point of this strict transform it suffices to show that the rank of the image in $\operatorname{Mat}_{d \times L}(k)$ of the matrix $\left(\left\langle a^{j}, m^{s}-n^{s}\right\rangle\right)_{1 \leq j \leq d, s \in L} \in \operatorname{Mat}_{d \times L}(\mathbf{Z})$ is $r$, which follows from the lemma.

PROPOSITION 6.3. If the regular fan $\Sigma$ with support $\check{\mathbf{R}}_{\geq 0}^{d}$ is compatible with all the hyperplanes $H_{m^{\ell}-n^{\ell}}$, the strict transform $X^{\prime}$ under the map $\pi(\Sigma): Z(\Sigma) \rightarrow$ $\mathbf{A}^{d}(k)$ of the subspace $X \subset \mathbf{A}^{d}(k)$ defined by the ideal of $k\left[u_{1}, \ldots, u_{d}\right]$ generated by the $\left(u^{m^{\ell}}-\lambda_{\ell} u^{n^{\ell}}\right)_{\ell \in\{1, \ldots, L\}}$ is regular and transversal to the exceptional divisor; it is also irreducible in each chart.

Proof. The preceding discussion shows that the rank of $J$ is $r$ everywhere on the strict transform of $X$, and by Zariski's jacobian criterion this strict transform is smooth and transversal to the exceptional divisor. But it is not necessarily irreducible; we show that the strict transform of our binomial variety is one of its irreducible components. Since the differences of the exponents in the total transform and the strict transform of a binomial are the same, the lattice of exponents generated by the exponents of all the strict transforms of the binomials $\left(u^{m^{\ell}}-\lambda_{m n} u^{n^{\ell}}\right)_{\ell \in\{1, \ldots, L\}}$ is the image $M(\sigma) \mathscr{L}$ of the lattice $\mathscr{L}$ by the linear map $\mathbf{Z}^{d} \rightarrow \mathbf{Z}^{d}$ corresponding to the matrix $M(\sigma)$ with rows $\left(a^{1}, \ldots, a^{d}\right)$. Similarly the exponents of the strict transforms of $u^{m^{1}}-\lambda_{m^{1} n^{1}} u^{n^{1}}, \ldots, u^{m^{r}}-\lambda_{m^{r} n^{r}} u^{n^{r}}$ generate the lattice $M(\sigma) \mathscr{L}_{1}$. The lattice $M(\sigma) \mathscr{L}$ is the saturation of $M(\sigma) \mathscr{L}_{1}$, and so according to [Eisenbud and Sturmfels 1996], since we assume that $k$ is algebraically closed, the strict transform of our binomial variety is one of the irreducible components of the binomial variety defined by the $r$ equations displayed above.

The charts corresponding to regular cones $\sigma \in \Sigma$ of dimension $<d$ are open subsets of those which we have just studied, so they contribute nothing new.

In the case of binomial varieties one can show that the regular refinement $\Sigma$ of the fan $\Sigma_{0}$ determined by the hyperplanes $H_{m^{s}-n^{s}}$ can be chosen in such a way that the restriction $X^{\prime} \rightarrow X$ of the map $\pi(\Sigma)$ to the strict transform $X^{\prime}$ of $X$ induces an isomorphism outside of the singular locus of $X$; it is therefore 
an embedded resolution of $X \subset \mathbf{A}^{d}(k)$ and not only a pseudoresolution; see [González Pérez and Teissier 2002] and [Teissier 2003, § 6.2].

REMARK. Since [Hironaka 1964], one usually seeks to achieve resolution of singularities by successions of blowing-ups with nonsingular centers, which moreover are "permissible". According to [De Concini and Procesi 1983; 1985], toric maps are dominated by finite successions of blowing-ups with nonsingular centers.

Now in view of the results of Section 5, we expect that if we deform a binomial variety by adding to each of its equations terms which do not affect the Newton polyhedron, the same toric map will resolve the deformed variety as well. However, it may be only a pseudoresolution, since the effect of the deformation on the singular locus is difficult to control. The next section shows that in a special case one can, conversely, present a singularity as a deformation of a toric variety, and thus obtain an embedded toric resolution.

\section{Resolution of Singularities of Branches}

This section is essentially an exposition of material in [Goldin and Teissier 2000] and [Teissier 2003]. The idea is to show that any analytically irreducible germ of curve is in a canonical way a deformation of a monomial curve, which is defined by binomial equations. In this terminological mishap, the monomial refers to the parametric presentation of the curve; the parametric presentation is more classical, but the binomial character of the equations is more suitable for resolution of singularities.

The deformation from the monomial curve to the curve is "equisingular", so that the toric map which resolves the singularties of the monomial curve according to Section 6 also resolves the singularities of our original curve once it is suitably embedded in the affine space where the monomial curve embeds. One interpretation of this is that after a suitable reembedding, any analytically irreducible curve becomes nondegenerate.

For example, in order to resolve the singularities at the origin of the plane curve $C$ with equation

$$
\left(u_{1}^{2}-u_{0}^{3}\right)^{2}-u_{0}^{5} u_{1}=0,
$$

a good method is to view it as the fiber for $v=1$ of the family of curves $C_{v}$ in $\mathbf{A}^{3}(k)$ defined by the equations

$$
\begin{aligned}
u_{1}^{2}-u_{0}^{3}-v u_{2} & =0, \\
u_{2}^{2}-u_{0}^{5} u_{1} & =0,
\end{aligned}
$$

as one can see by eliminating $u_{2}$ between the two equations. The advantage is that the fiber for $v=0$ is a binomial variety, which we know how to resolve, and its resolution also resolves all the fibers $C_{v}$. For $v \neq 0$, the fiber $C_{v}$ is isomorphic to our original plane curve $C$, re-embedded in $\mathbf{A}^{3}(k)$ by the functions $u_{0}, u_{1}, u_{1}^{2}-u_{0}^{3}$. 
In more algebraic terms, it gives this:

Let $R$ be a one dimensional excellent equicharacteristic local ring whose completion is an integral domain and whose residue field is algebraically closed. A basic example is $R=k \llbracket x, y \rrbracket /(f)$ where $k$ is algebraically closed and $f(x, y)$ is irreducible in $k \llbracket x, y \rrbracket$. Then the normalization $\bar{R}$ of $R$ is a (discrete) valuation ring because it is a one dimensional normal local ring. The maximal ideal of $\bar{R}$ is generated by a single element, say $t$, and each nonzero element of $\bar{R}$ can be written uniquely as $u t^{n}$, where $u$ is invertible in $\bar{R}$ and $n \in \mathbf{N} \cup\{0\}$. The valuation $\nu\left(u t^{n}\right)$ of that element is $n$.

In our basic example, the inclusion $R \subset \bar{R}$ is $k \llbracket x, y \rrbracket /(f) \subset k \llbracket t \rrbracket$ given by $x \mapsto x(t), y \mapsto y(t)$, where $x(t), y(t)$ is a parametrization of the plane curve with equation $f(x, y)=0$.

Since $R$ is a subalgebra of $\bar{R}$, the values taken by the valuation on the elements of $R$ (except 0 ) form a semigroup $\Gamma$ contained in $\mathbf{N}$. This semigroup has a finite complement in $\mathbf{N}$ and is finitely generated. Let us write it

$$
\Gamma=\left\langle\gamma_{0}, \gamma_{1}, \ldots, \gamma_{g}\right\rangle
$$

The powers of the maximal ideal of $\bar{R}$ form a filtration

$$
\bar{R} \supset t \bar{R} \supset t^{2} \bar{R} \supset \cdots \supset t^{n} \bar{R} \supset \cdots
$$

whose associated graded ring

$$
\operatorname{gr}_{\nu} \bar{R}=\bigoplus_{n \in \mathbf{N} \cup\{0\}} t^{n} \bar{R} / t^{n+1} \bar{R}
$$

is a $k$-algebra isomorphic to the polynomial $\operatorname{ring} k[t]$ by the map $t\left(\bmod t^{2} \bar{R}\right) \mapsto t$.

This filtration induces a filtration on the $\operatorname{ring} R$ itself, by the ideals $\mathscr{P}_{n}=$ $R \cap t^{n} \bar{R}$, and one defines the corresponding associated graded ring

$$
\operatorname{gr}_{\nu} R=\bigoplus_{n \in \mathrm{N} \cup\{0\}} \mathscr{P}_{n} / \mathscr{P}_{n+1} \subseteq \operatorname{gr}_{\nu} \bar{R}=k[t] .
$$

Proposition 7.1 [Goldin and Teissier 2000]. The subalgebra $\operatorname{gr}_{\nu} R$ of $k[t]$ is equal to the subalgebra generated by $t^{\gamma_{0}}, t^{\gamma_{1}}, \ldots, t^{\gamma_{g}}$. It is the semigroup algebra over $k$ of the semigroup $\Gamma$ of the valuation $\nu$ on $R$; it is also the affine algebra of the monomial curve in the affine space $\mathbf{A}^{g+1}(k)$ described parametrically by $u_{i}=t^{\gamma_{i}}$ for $0 \leq i \leq g$.

There is a precise geometrical relationship between the original curve $C$ with algebra $R$ and the monomial curve $C^{\Gamma}$ with algebra $\operatorname{gr}_{\nu} R$ : according to a general principle of algebra, the $\operatorname{ring} R$ is a deformation of its associated graded ring. More precisely, assume that $R$ contain a field of representatives of its residue field $k$, i.e., that we have a subfield $k \subset R$ such that the composed map $k \subset$ $R \rightarrow R / m=k$ is the identity. This will be the case in particular, according 
to Cohen's theorem, if the local ring $R$ is complete (and equicharacteristic of course).

Start from the filtration by the ideals $\mathscr{P}_{n}$ introduced above, set $\mathscr{P}_{n}=R$ for $n \leq 0$ and consider the algebra

$$
\mathscr{A}_{\nu}(R)=\bigoplus_{n \in \mathbf{Z}} \mathscr{P}_{n} v^{-n} \subset R\left[v, v^{-1}\right] .
$$

It can be shown (see [Teissier 2003]) that it is generated as a $R[v]$-algebra by the $\xi_{i} v^{-\gamma_{i}}, 0 \leq i \leq g$, where $\xi_{i} \in R$ is of $t$-adic order $\gamma_{i}$. Since $\mathscr{P}_{n}=R$ for $n \leq 0$ it contains as a graded subalgebra the polynomial algebra $R[v]$, and therefore also $k[v]$.

Proposition 7.2 [Teissier 1975; Bourbaki 1983, Ch. VIII $\S 6$, exerc. 2]; see also [Gerstenhaber 1964; 1966].

(a) The composed map $k[v] \rightarrow \mathscr{A}_{\nu}(R)$ is faithfully flat.

(b) The map

$$
\sum x_{n} v^{-n} \mapsto \sum \bar{x}_{n}
$$

where $\bar{x}_{n}$ is the image of $x_{n}$ in the quotient $\mathscr{P}_{n} / \mathscr{P}_{n+1}$, induces an isomorphism

$$
\mathscr{A}_{\nu}(R) / v \mathscr{A}_{\nu}(R) \rightarrow \operatorname{gr}_{\nu} R
$$

(c) For any $v_{0} \in k^{*}$ the map

$$
\sum x_{n} v^{-n} \mapsto \sum x_{n} v_{0}^{-n}
$$

induces an isomorphism of $k$-algebras

$$
\mathscr{A}_{\nu}(R) /\left(v-v_{0}\right) \mathscr{A}_{\nu}(R) \rightarrow R .
$$

Proof. Since $k[v]$ is a principal ideal domain, to prove (a) it suffices by [Bourbaki 1968 , Ch. I $\S 3.1]$ to prove that $\mathscr{A}_{\nu}(R)$ has no torsion as a $k[v]$-module and that for any $v_{0} \in k$ we have $\left(v-v_{0}\right) \mathscr{A}_{\nu}(R) \neq \mathscr{A}_{\nu}(R)$. The second statement follows from (b) and (c), which are easy to verify, and the first follows from the fact that $\mathscr{A}_{\nu}(R)$ is a subalgebra of $R\left[v, v^{-1}\right]$.

This proposition means that there is a one parameter flat family of algebras whose special fiber is the graded algebra and all other fibers are isomorphic to $R$. Geometrically, this gives us a flat family of curves whose special fiber is the monomial curve and all other fibers are isomorphic to our given curve. This deformation can be realized in the following way. I assume for simplicity that $R$ is complete. Then by the definition of the semigroup $\Gamma$ there are elements $\xi_{0}(t), \ldots, \xi_{g}(t)$ in $k \llbracket t \rrbracket$ that belong to $R$ and are such that their $t$-adic valuations are the generators $\gamma_{i}$ of the semigroup $\Gamma$. We may write $\xi_{i}(t)=$ 
$t^{\gamma_{i}}+\sum_{j>\gamma_{i}} b_{i j} t^{j}$ with $b_{i j} \in k$. Now introduce a parameter $v$ and consider the family of parametrized curves in $\mathbf{A}^{g+1}(k)$ described as follows:

$$
\begin{aligned}
& u_{0}=\xi_{0}(v t) v^{-\gamma_{0}}=t^{\gamma_{0}}+\sum_{j>\gamma_{0}} b_{0 j} v^{j-\gamma_{0}} t^{j}, \\
& u_{1}=\xi_{1}(v t) v^{-\gamma_{1}}=t^{\gamma_{1}}+\sum_{j>\gamma_{1}} b_{1 j} v^{j-\gamma_{1}} t^{j}, \\
& \ldots \ldots \ldots \ldots \ldots \cdots \cdots \cdots \cdots \cdots \cdots \cdots \cdots \cdots \cdots \cdots \cdots \cdots \cdots \cdots \\
& u_{g}=\xi_{g}(v t) v^{-\gamma_{g}}=t^{\gamma_{g}}+\sum_{j>\gamma_{g}} b_{g j} v^{j-\gamma_{g}} t^{j} .
\end{aligned}
$$

The parametrization shows that for $v=0$ we obtain the monomial curve, and for any $v \neq 0$ a curve isomorphic to our given curve, as embedded in $\mathbf{A}^{g+1}(k)$ by the functions $\xi_{0}, \ldots, \xi_{g}$. This is a realisation of the family of Proposition 7.2. In order to get an equational representation of that family, we must begin by finding the equations of the monomial curve, which we will then proceed to deform.

The equations of the monomial curve $C^{\Gamma}$ correspond to the relations between the generators $\gamma_{i}$ of $\Gamma$. They are fairly simple in the case where $\Gamma$ is the semigroup of a plane branch, and in that case $C^{\Gamma}$ is a complete intersection. The general setup is as follows:

Consider the $\mathbf{Z}$-linear map $w: \mathbf{Z}^{g+1} \rightarrow \mathbf{Z}$ determined by sending the $i$-th base vector $e_{i}$ to $\gamma_{i}$; the image of $\mathbf{Z}_{\geq 0}^{g+1}$ is $\Gamma$. It is not difficult to see that the kernel of $w$ is generated by differences $m-m^{\prime}$, where $m, m^{\prime} \in \mathbf{Z}_{\geq 0}^{g+1}$ and $w(m)=w\left(m^{\prime}\right)$. The kernel of $w$ is a lattice (free sub $\mathbf{Z}$-module) $\mathscr{L}$ in $\mathbf{Z}^{g+1}$, which must be finitely generated because $\mathbf{Z}^{g+1}$ is a noetherian $\mathbf{Z}$-module and $\mathbf{Z}$ is a principal ideal domain.

If we choose a basis $m^{1}-n^{1}, \ldots, m^{q}-n^{q}$ for $\mathscr{L}$, such that all the $m^{j}, n^{j}$ are in $\mathbf{Z}_{\geq 0}^{g+1}$, it follows from the very construction of semigroup algebras that $C^{\Gamma}$ is defined in the space $\mathbf{A}^{g+1}(k)$ with coordinates $u_{0}, \ldots, u_{g}$ by the vanishing of the binomials $u^{m^{1}}-u^{n^{1}}, \ldots, u^{m^{q}}-u^{n^{q}}$.

Now the faithful flatness of the family of Proposition 7.2 implies that it can be defined in $\mathbf{A}^{1}(k) \times \mathbf{A}^{g+1}(k)$ by equations which are deformations of the equations of the monomial curve [Teissier 2003, $\S 5$, proof of 5.49]. Here I cheat a little by leaving out the fact that one in fact defines a formal space. Anyway, our family of (formal) curves is also defined by equations of the form

$$
\begin{aligned}
& u^{m^{1}}-u^{n^{1}}+\sum_{w(r)>w\left(m^{1}\right)} c_{r}^{(1)}(v) u^{r}=0, \\
& u^{m^{2}}-u^{n^{2}}+\sum_{w(r)>w\left(m^{2}\right)} c_{r}^{(2)}(v) u^{r}=0, \\
& \ldots \ldots \ldots \ldots \cdots \cdots \cdots \cdots \cdots \cdots \cdots \cdots \cdots \cdots \cdots \cdots \\
& u^{m^{q}}-u^{n^{q}}+\sum_{w(r)>w\left(m^{q}\right)} c_{r}^{(1)}(v) u^{r}=0,
\end{aligned}
$$


where the $c_{r}^{(j)}(v)$ are in $(v) k \llbracket v \rrbracket, w(r)=\sum_{0}^{g} \gamma_{j} r_{j}$ is the weight of the monomial $u^{r}$ with respect to the weight vector $w=\left(\gamma_{0}, \ldots, \gamma_{g}\right)$, that is, $w(r)=\langle w, r\rangle$. Remember that by construction $w\left(m^{i}\right)=w\left(n^{i}\right)$ for $1 \leq i \leq q$. This means that we deform each binomial equations by adding terms of weight greater than that of the binomial. It is shown in [Teissier 2003] that the parametric representation and the equation representation both describe the deformation of Proposition 7.2. Up to completion with respect to the $\left(u_{0}, \ldots, u_{g}\right)$-adic topology, the algebra $\mathscr{A}_{\nu}(R)$ is the quotient of $k[v] \llbracket u_{0}, \ldots, u_{g} \rrbracket$ by the ideal generated by the equations written above. It is also equal to the subalgebra $k \llbracket \xi_{0}(v t) v^{-\gamma_{0}}, \ldots, \xi_{g}(v t) v^{-\gamma_{g}} \rrbracket$ of $k[v] \llbracket t \rrbracket$.

One may remark that, in the case where the $\xi_{j}(t)$ are polynomials, there is a close analogy with the SAGBI algebras bases for the subalgebra $k\left[\xi_{0}(t), \xi_{1}(t)\right] \subset$ $k[t]$ (see [Sturmfels 1996]). This is developed in [Bravo 2004].

This equation description is the generalization of the example shown at the beginning of this section.

Now it should be more or less a computational exercise to check that a toric map $Z(\Sigma) \rightarrow \mathbf{A}^{g+1}$ which resolves the binomial variety $C^{\Gamma}$ also resolves the "nearby fibers", which are all isomorphic to $C$ re-embedded in $\mathbf{A}^{g+1}$. There is however a difficulty [Goldin and Teissier 2000] which requires the use of Zariski's main theorem.

The results of this section have been extended in [González Pérez 2003] to the much wider class of irreducible quasi-ordinary germs of hypersurface singularities, whose singularities are not isolated in general.

This shows that a toric resolution of binomial varieties can be used, by considering suitable deformations, to resolve singularities which are at first sight far from binomial.

\section{Appendix: Multiplicities, Volumes and Nondegeneracy}

Multiplicities and volumes. One of the interesting features of the BriançonSkoda theorem is that it provides a way to pass from the integral closure of an ideal to the ideal itself, while it is much easier to check that a given element is in the integral closure of an ideal than to check that it is in the ideal. For this reason, the theorem has important applications in problems of effective commutative algebra motivated by transcendental number theory. In the same vein, this section deals, in the monomial case, with the problem of determining from numerical invariants whether two ideals have the same integral closure, which is much easier than to determine whether they are equal. The basic fact coming to light is that multiplicities in commutative algebra are like volumes in the theory of convex bodies, and indeed, for monomial ideals, they are volumes, up to a factor of $d$ ! (compare with [Teissier 1988]). The same is true for degrees of invertible sheaves on algebraic varieties. Exactly as monomial ideals, and for the same reason, the degrees of equivariant invertible sheaves generated by 
their global sections on toric varieties are volumes of compact convex bodies multiplied by $d$ ! [Teissier 1979].

In this appendix proofs are essentially replaced by references; for the next four paragraphs, see [Bourbaki 1983, Ch. VIII, § 4].

Let $R$ be a noetherian ring and $\mathfrak{q}$ an ideal of $R$ such that the $R$-module $R / \mathfrak{q}$ has finite length $\ell_{R}(R / \mathfrak{q})=\ell_{R / \mathfrak{q}}(R / \mathfrak{q})$. Then the quotients $\mathfrak{q}^{n} / \mathfrak{q}^{n+1}$ have finite length as $R / \mathfrak{q}$-modules and one can define the Hilbert-Samuel series

$$
H_{R, \mathfrak{q}}=\sum_{n=0}^{\infty} \ell_{R / \mathfrak{q}}\left(\mathfrak{q}^{n} / \mathfrak{q}^{n+1}\right) T^{n} \in \mathbf{Z} \llbracket T \rrbracket .
$$

There exist an integer $d \geq 0$ and an element $P \in \mathbf{Z}\left[T, T^{-1}\right]$ such that $P(1)>0$ and

$$
H_{R, \mathfrak{q}}=(1-T)^{-d} P \text {. }
$$

From this follows:

Proposition A.1 (Samuel). Given $R$ and $\mathfrak{q}$ as above, there exist an integer $N_{0}$ and a polynomial $Q(U)$ with rational coefficients such that for $n \geq N_{0}$ we have

$$
\ell_{R / \mathfrak{q}}\left(R / \mathfrak{q}^{n}\right)=Q(n) .
$$

If we assume that $\mathfrak{q}$ is primary for some maximal ideal $m$ of $R$, i.e., $\mathfrak{q} \supset m^{k}$ for large enough $k$, the degree of the polynomial $Q$ is the dimension $d$ of the local ring $R_{m}$, and the highest degree term of $Q(U)$ can be written $e(\mathfrak{q}, R) U^{d} / d$ !. In fact, $e(\mathfrak{q}, R)=P(1) \in \mathbf{N}$.

By definition, the integer $e(\mathfrak{q}, R)$ is the multiplicity of the ideal $\mathfrak{q}$ in $R$.

If $R$ contains a field $k$ such that $k=R / m$, we can replace $\ell_{R / \mathfrak{q}}\left(R / \mathfrak{q}^{n}\right)$ by its dimension $\operatorname{dim}_{k}\left(R / \mathfrak{q}^{n}\right)$ as a $k$-vector space.

Take $R=k\left[u_{1}, \ldots, u_{d}\right]$ and $\mathfrak{q}=\left(u^{m^{1}}, \ldots, u^{m^{q}}\right) R$; the ideal $\mathfrak{q}$ is primary for the maximal ideal $m=\left(u_{1}, \ldots, u_{d}\right) R$ if and only if $\operatorname{dim}_{k} R / \mathfrak{q}<\infty$. Now one sees that the images of the monomials $u^{m}$ such that $m$ is not contained in $E=\bigcup_{i=1}^{q}\left(m^{i}+\mathbf{R}_{\geq 0}^{d}\right)$ constitute a basis of the $k$-vector space $R / \mathfrak{q}$ :

$$
\operatorname{dim}_{k} R / \mathfrak{q}=\# \mathbf{Z}^{d} \cap\left(\mathbf{R}_{\geq 0}^{d} \backslash E\right) .
$$

For the same reason we have for all $n \geq 1$, since $\mathfrak{q}^{n}$ is also monomial,

$$
\operatorname{dim}_{k} R / \mathfrak{q}^{n}=\# \mathbf{Z}^{d} \cap\left(\mathbf{R}_{\geq 0}^{d} \backslash n E\right),
$$

where $n E$ is the set of sums of $n$ elements of $E$.

From this follows, in view of the polynomial character of the first term of the equality:

Corollary A.2. Given a subset $E=\bigcup_{s=1}^{q}\left(m^{s}+\mathbf{R}_{\geq 0}^{d}\right)$ whose complement in $\mathbf{R}_{\geq 0}^{d}$ has finite volume, there exists an integer $N_{0}$ and a polynomial $Q(n)$ of degree $d$ with rational coefficients such that for $n \geq N_{0}$ we have

$$
\# \mathbf{Z}^{d} \cap\left(\mathbf{R}_{\geq 0}^{d} \backslash n E\right)=Q(n) .
$$


Therefore,

$$
\lim _{n \rightarrow \infty} \frac{Q(n)}{n^{d}}=\lim _{n \rightarrow \infty} \frac{\# \mathbf{Z}^{d} \cap\left(\mathbf{R}_{\geq 0}^{d} \backslash n E\right)}{n^{d}}=\lim _{n \rightarrow \infty} \operatorname{Covol} \frac{n E}{n}=\operatorname{Covol} \bar{E},
$$

where Covol $A$, the covolume of $A$, is the volume of the complement of $A$ in $\mathbf{R}_{\geq 0}^{d}$. The last equality follows from the remark made in Section 4 , and the previous one from the classical fact of calculus that as $n \rightarrow \infty$,

$$
\text { Covol } \frac{n E}{n}=\frac{\# \mathbf{Z}^{d} \cap\left(\mathbf{R}_{\geq 0}^{d} \backslash n E\right)}{n^{d}}+o(1) .
$$

Since the limit as $n \rightarrow \infty$ of $Q(n) / n^{d}$ is $e(\mathfrak{q}, R) / d$ !, we have immediately:

Corollary A.3. For a monomial ideal $\mathfrak{q}=\left(u^{m^{1}}, \ldots, u^{m^{s}}\right)$ in $R=k\left[u_{1}, \ldots, u_{d}\right]$ which is primary for $m=\left(u_{1}, \ldots, u_{d}\right)$, with the notations above, we have

$$
\begin{aligned}
\operatorname{dim}_{k}(R / \mathfrak{q}) & =\# \mathbf{Z}^{d} \cap\left(\mathbf{R}_{\geq 0}^{d} \backslash E\right), \\
e(\mathfrak{q}, R) & =d ! \text { Covol } \bar{E} .
\end{aligned}
$$

Corollary A.4 (Monomial Rees Theorem, an avatar of [Rees 1961]).

(a) For a monomial primary ideal $\mathfrak{q}$ as above, me have

$$
e(\overline{\mathfrak{q}}, R)=e(\mathfrak{q}, R) .
$$

(b) Given two such ideals $\mathfrak{q}_{1}, \mathfrak{q}_{2}$ such that $\mathfrak{q}_{1} \subseteq \mathfrak{q}_{2}$, we have $\overline{\mathfrak{q}}_{1}=\overline{\mathfrak{q}}_{2}$ if and only if $e\left(\mathfrak{q}_{1}, R\right)=e\left(\mathfrak{q}_{2}, R\right)$.

These results hold for ideals containing a power of the maximal ideal in a noetherian local ring $R$ whose completion is equidimensional [Rees 1961].

Now there is a well-known theorem in the theory of convex bodies, concerning the volume of the Minkowski sum of compact convex sets. Recall that for $K_{1}, K_{2}$ in $\mathbf{R}^{d}$, the Minkowski sum $K_{1}+K_{2}$ is the set of sums $\left\{x_{1}+x_{2}: x_{1} \in K_{1}, x_{2} \in\right.$ $\left.K_{2}\right\}$; also we set $\lambda K=\{\lambda x: x \in K\}$ for $\lambda \in \mathbf{R}$. Then:

Theorem A.5 (Minkowski). Given s compact convex subsets $K_{1}, \ldots, K_{s}$ of $\mathbf{R}^{d}$, there is a homogeneous expression for the d-dimensional volume of the positive Minkowski linear combination of the $K_{i}$, with $\left(\lambda_{i}\right)_{1 \leq i \leq s} \in \mathbf{R}_{\geq 0}^{s}$ :

$$
\operatorname{Vol}_{d}\left(\lambda_{1} K_{1}+\cdots+\lambda_{s} K_{s}\right)=\sum_{\sum_{1}^{s} \alpha_{i}=d} \frac{d !}{\alpha_{1} ! \ldots \alpha_{s} !} \operatorname{Vol}\left(K_{1}^{\left[\alpha_{1}\right]}, \ldots, K_{s}^{\left[\alpha_{s}\right]}\right) \lambda_{1}^{\alpha_{1}} \ldots \lambda_{s}^{\alpha_{s}}
$$

where the coefficients $\operatorname{Vol}\left(K_{1}^{\left[\alpha_{1}\right]}, \ldots, K_{s}^{\left[\alpha_{s}\right]}\right)$ are nonnegative and are called the mixed volumes of the convex sets $K_{i}$.

In particular, with $s=2$,

$$
\operatorname{Vol}_{d}\left(\lambda_{1} K_{1}+\lambda_{2} K_{2}\right)=\sum_{i=0}^{d}\left(\begin{array}{c}
d \\
i
\end{array}\right) \operatorname{Vol}\left(K_{1}^{[i]}, K_{2}^{[d-i]}\right) \lambda_{1}^{i} \lambda_{2}^{d-i} .
$$


The proof is obtained by approximating the convex bodies by polytopes, and using the Cauchy formula for the volume of polytopes. Exactly the same proof applies to the covolumes of convex subsets of $\mathbf{R}_{\geq 0}^{d}$ to give the corresponding theorem:

$\operatorname{Covol}_{d}\left(\lambda_{1} \bar{E}_{1}+\cdots+\lambda_{s} \bar{E}_{s}\right)=\sum_{\sum_{1}^{s} \alpha_{i}=d} \frac{d !}{\alpha_{1} ! \ldots \alpha_{s} !} \operatorname{Covol}\left(\bar{E}_{1}^{\left[\alpha_{1}\right]}, \ldots, \bar{E}_{s}^{\left[\alpha_{s}\right]}\right) \lambda_{1}^{\alpha_{1}} \ldots \lambda_{s}^{\alpha_{s}}$, defining the mixed covolumes of such convex subsets.

There is an analogous formula in commutative algebra:

Theorem A.6 [Teissier 1973]. Given ideals $\mathfrak{q}_{1}, \ldots, \mathfrak{q}_{s}$ which are primary for a maximal ideal $m$ in a noetherian ring $R$ such that the localization $R_{m}$ is a $d$-dimensional local ring and the residue field $R_{m} / m R_{m}$ is infinite, there is for $\lambda_{1}, \ldots, \lambda_{s} \in \mathbf{Z}_{\geq 0}^{s}$ an expression

$$
e\left(\mathfrak{q}_{1}^{\lambda_{1}} \ldots \mathfrak{q}_{s}^{\lambda_{s}}, R\right)=\sum_{\sum_{1}^{s} \alpha_{i}=d} \frac{d !}{\alpha_{1} ! \ldots \alpha_{s} !} e\left(\mathfrak{q}_{1}^{\left[\alpha_{1}\right]}, \ldots, \mathfrak{q}_{s}^{\left[\alpha_{s}\right]} ; R\right) \lambda_{1}^{\alpha_{1}} \ldots \lambda_{s}^{\alpha_{s}},
$$

where the coefficients $e\left(\mathfrak{q}_{1}^{\left[\alpha_{1}\right]}, \ldots, \mathfrak{q}_{s}^{\left[\alpha_{s}\right]} ; R\right)$ are nonnegative integers and are called the mixed multiplicities of the primary ideals $\mathfrak{q}_{i}$. (This name is justified by the fact that $e\left(\mathfrak{q}_{1}^{\left[\alpha_{1}\right]}, \ldots, \mathfrak{q}_{s}^{\left[\alpha_{s}\right]} ; R\right)$ is the multiplicity of an ideal generated by $\alpha_{1}$ elements of $\mathfrak{q}_{1}, \ldots, \alpha_{s}$ elements of $\mathfrak{q}_{s}$, chosen in a sufficiently general way.) Taking $s=2$ gives

$$
e\left(\mathfrak{q}_{1}^{\lambda_{1}} \mathfrak{q}_{2}^{\lambda_{2}}, R\right)=\sum_{i=0}^{d}\left(\begin{array}{c}
d \\
i
\end{array}\right) e\left(\mathfrak{q}_{1}^{[i]}, \mathfrak{q}_{2}^{[d-i]} ; R\right) \lambda_{1}^{i} \lambda_{2}^{d-i} .
$$

From this and Corollary A.3 there follows immediately:

Corollary A.7. Let $k$ be an infinite field. Given monomial ideals $\mathfrak{q}_{1}, \ldots, \mathfrak{q}_{s}$ which are primary for the maximal ideal $\left(u_{1}, \ldots, u_{d}\right)$ in $R=k\left[u_{1}, \ldots, u_{d}\right]$, and denoting by $E_{i}$ the corresponding subsets generated by their exponents, we have for all $\alpha \in \mathbf{Z}_{\geq 0}^{s}$ such $\sum_{1}^{s} \alpha_{i}=d$ the equality

$$
e\left(\mathfrak{q}_{1}^{\left[\alpha_{1}\right]}, \ldots, \mathfrak{q}_{s}^{\left[\alpha_{s}\right]} ; R\right)=d ! \operatorname{Covol}\left(\bar{E}_{1}^{\left[\alpha_{1}\right]}, \ldots, \bar{E}_{s}^{\left[\alpha_{s}\right]}\right) .
$$

In particular, the mixed multiplicities depend only on the integral closures of the ideals $\mathfrak{q}_{i}$. Now we have the well-known Alexandrov-Fenchel inequalities for the mixed volumes of two compact convex bodies:

Theorem A.8 (Alexandrov and Fenchel; see [Gromov 1990]).

(a) Let $K_{1}, K_{2}$ be compact convex bodies in $\mathbf{R}^{d}$; set $v_{i}=\operatorname{Vol}\left(K_{1}^{[i]}, K_{2}^{[d-i]}\right)$. For all $2 \leq i \leq d$,

$$
v_{i-1}^{2} \geq v_{i} v_{i-2}
$$


(b) Equality holds in all these inequalities if and only if for some $\rho \in \mathbf{R}_{+}$we have $K_{1}=\rho K_{2}$ up to translation. If all the $v_{i}$ are equal, then $K_{1}=K_{2}$ up to translation, and conversely.

Let $\mathbf{B}^{d}$ denote the $d$-dimensional unit ball, and $A$ any subset of $\mathbf{R}^{d}$ which is tame enough for the volumes to exist.

The problem that inspired this theorem is to prove that in the isoperimetric inequality $\operatorname{Vol}_{d-1}(\partial A)^{d} \geq d^{d} \operatorname{Vol}_{d}\left(\mathbf{B}^{d}\right) \operatorname{Vol}_{d}(A)^{d-1}$, equality should hold only if $A$ is a multiple of the unit ball, to which some "hairs" of a smaller dimension than $\partial A$ have been added. In the case where $A$ is convex, taking $K_{1}$ to be the unit ball and $K_{2}=A$, one notices that $v_{0}=\operatorname{Vol}_{d}(A)$ and $v_{1}=d^{-1} \operatorname{Vol}_{d-1}(\partial A)$; the isoperimetric inequality then follows very quickly by an appropriate telescoping of the Alexandrov-Fenchel inequalities. From this telescoping follows the fact that if we have equality in the isoperimetric inequality for a convex subset $A$ of $\mathbf{R}^{d}$, then we have equality in all the Alexandrov-Fenchel inequalities for $A$ and the unit ball, so that $A$ must be a ball. By the same type of telescoping, one proves the inequalities $v_{i}^{d} \geq v_{0}^{d-i} v_{d}^{i}$, which yields:

Theorem A.9 (BRÜnN And Minkowski; see [Gromov 1990]). For convex compact subsets $K_{1}, K_{2}$ of $\mathbf{R}^{d}$,

$$
\operatorname{Vol}_{d}\left(K_{1}+K_{2}\right)^{1 / d} \geq \operatorname{Vol}_{d}\left(K_{1}\right)^{1 / d}+\operatorname{Vol}_{d}\left(K_{2}\right)^{1 / d} .
$$

Equality holds if and only if the two sets are homothetic up to translation, or one of them is a point, or $\operatorname{Vol}_{d}\left(K_{1}+K_{2}\right)^{1 / d}=0$.

The same constructions and proof apply to covolumes, where the inequalities are reversed; they correspond to inequalities for the mixed multiplicities of monomial ideals, which are in fact true for primary ideals in formally equidimensional noetherian local rings:

Theorem A.10 [Teissier 1977; 1978; Rees and Sharp 1978; Katz 1988]. Let $\mathfrak{q}_{1}, \mathfrak{q}_{2}$ be primary ideals in the $d$-dimensional noetherian local ring $R$. Set

$$
w_{i}=e\left(\mathfrak{q}_{1}^{[i]}, \mathfrak{q}_{2}^{[d-i]} ; R\right) .
$$

(a) We have $w_{i-1}^{2} \leq w_{i} w_{i-2}$ for $2 \leq i \leq d$.

(b) The inequalities $e\left(\mathfrak{q}_{1} \mathfrak{q}_{2}, R\right)^{1 / d} \leq e\left(\mathfrak{q}_{1}, R\right)^{1 / d}+e\left(\mathfrak{q}_{2}, R\right)^{1 / d}$ hold, with equality if and only if the inequalities of $(a)$ are equalities.

(c) Assuming in addition that $R$ is formally equidimensional (quasi-unmixed), equality holds in all these inequalities if and only if $\overline{\mathfrak{q}_{1}^{a}}=\overline{\mathfrak{q}_{2}^{b}}$ for some $a, b \in \mathbf{N}$. If all the $w_{i}$ are equal, then $\overline{\mathfrak{q}}_{1}=\overline{\mathfrak{q}}_{2}$, and conversely.

So in this case again, the combinatorial inequalities appear as the avatar for monomial ideals of general inequalities of commutative algebra. One can see that if $\mathfrak{q}_{1} \subseteq \mathfrak{q}_{2}$, we have $e\left(\mathfrak{q}_{1}, R\right)=w_{d} \geq w_{i} \geq w_{0}=e\left(\mathfrak{q}_{2}, R\right)$, for $1 \leq i \leq d-1$. So this result implies Rees' Theorem, which is stated after Corollary A.4. 
In fact the same happens for the Alexandrov-Fenchel inequalities, which are the avatars for toric varieties associated to polytopes of general inequalities of Kähler geometry known as the Hodge Index Theorem. This is because the mixed volumes of rational convex polytopes are equal, up to a $d$ ! factor, to the mixed degrees of invertible sheaves (or of divisors) on certain toric varieties associated to the collection of polytopes, exactly as in Corollary A.7. This approach to Alexandrov-Fenchel inequalities was introduced by Khovanskii and the author; see [Gromov 1990] for an excellent exposition of this topic, and [Khovanskii 1979; Teissier 1979].

In all these cases, it is remarkable that, thanks to the positivity and convexity properties of volumes and of multiplicities, a finite number of equations on a pair $\left(A_{1}, A_{2}\right)$ of objects in an infinite dimensional space (convex bodies modulo translation or integrally closed primary ideals) suffices to ensure that $A_{1}=A_{2}$.

Newton nondegenerate ideals in $k \llbracket u_{1}, \ldots, u_{d} \rrbracket$ and multiplicities. Define the support $S(I)$ of an ideal $I$ of $k \llbracket u_{1}, \ldots, u_{d} \rrbracket$ to be the set of the exponents $m$ appearing as one of the exponents in at least one series belonging to the ideal $I$. Define the Newton polyhedron $\mathscr{N}_{+}(I)$ of $I$ as the boundary of the convex hull $\mathscr{P}_{+}(I)$ of $\bigcup_{m \in S(I)}\left(m+\mathbf{R}_{\geq 0}^{d}\right)$.

According to [Bivià-Ausina et al. 2002], a primary ideal $\mathfrak{q}$ is said to be nondegenerate if it admits a system of generators $q_{1}, \ldots, q_{t}$ such that their restrictions to each compact face of $\mathscr{N}_{+}(I)$ have no common zero in the torus $\left(k^{*}\right)^{d}$. The following is part of what is proved in [Bivià-Ausina et al. 2002, §3]:

TheOREM A.11. For a primary ideal $\mathfrak{q}$ of $R=k \llbracket u_{1}, \ldots, u_{d} \rrbracket$, the following conditions are equivalent:

(a) The ideal $\mathfrak{q}$ is nondegenerate in the coordinates $u_{1}, \ldots, u_{d}$.

(b) $e(\mathfrak{q}, R)=d ! \operatorname{Covol} \mathscr{P}_{+}(I)$.

(c) The integral closure $\overline{\mathfrak{q}}$ of $\mathfrak{q}$ is generated by monomials in $u_{1}, \ldots, u_{d}$.

It follows from this that monomial ideals are nondegenerate, and that products of nondegenerate primary ideals are nondegenerate [Bivià-Ausina et al. 2002, Corollary 3.14]. Moreover, all the numerical facts mentioned above for monomial ideals with respect to their Newton polyhedron are valid for nondegenerate ideals (loc. cit.). Nondegenerate ideals behave as reductions of monomial ideals, which in fact they are. Here we can think of a reduction (in the sense of [Northcott and Rees 1954]; see also see [Rees 1984]) of an ideal $\mathscr{M} \subset k \llbracket u_{1}, \ldots, u_{d} \rrbracket$ as an ideal generated by $d$ sufficiently general combinations of generators of $\mathscr{M}$. More precisely, it is an ideal $\mathscr{M}^{\prime}$ contained in $\mathscr{M}$ and having the same integral closure.

There is a close connection between this nondegeneracy for ideals and the results of section 5 ; if the ideal $\mathfrak{q}=\left(q_{1}, \ldots, q_{s}\right) k \llbracket u_{1}, \ldots, u_{d} \rrbracket$ is nondegenerate, then a general linear combination $q=\sum_{i=1}^{s} \lambda_{i} q_{i}$ is nondegenerate with respect to its Newton polyhedron. 
There are many other interesting consequences of the relationship between monomial ideals and combinatorics; I refer the reader to [Sturmfels 1996].

All the results of this appendix remain valid if $k\left[u_{1}, \ldots, u_{d}\right]$ and its completion $k \llbracket u_{1}, \ldots, u_{d} \rrbracket$ are replaced respectively by $k\left[\check{\sigma} \cap \mathbf{Z}^{d}\right]$ and its completion, for a strictly convex cone $\sigma \subset \mathbf{R}_{\geq 0}^{d}$.

There are also generalizations of mixed multiplicities to collections of not necessarily primary ideals [Rees 1986] and to the case where one of the ideals is replaced by a submodule of finite colength of a free $R$-module of finite type [Kleiman and Thorup 1996].

It would be interesting to determine how the results of this appendix extend to monomial submodules of a free $k\left[u_{1}, \ldots, u_{d}\right]$-module.

\section{References}

[Bivià-Ausina et al. 2002] C. Bivià-Ausina, T. Fukui, and M. J. Saia, "Newton filtrations, graded algebras and codimension of non-degenerate ideals", Math. Proc. Cambridge Philos. Soc. 133:1 (2002), 55-75.

[Blickle and Lazarsfeld 2004] M. Blickle and R. Lazarsfeld, "An informal introduction to multiplier ideals", pp. 87-114 in Trends in algebraic geometry, edited by L. Avramov et al., Math. Sci. Res. Inst. Publ. 51, Cambridge University Press, New York, 2004.

[Bourbaki 1968] N. Bourbaki, Algèbre commutative, Ch. I à IV, Masson, Paris, 1968.

[Bourbaki 1983] N. Bourbaki, Algèbre commutative, Ch. VIII et IX, Masson, Paris, 1983.

[Bravo 2004] A. Bravo, "Some facts about canonical subalgebra bases", pp. 247-254 in Trends in algebraic geometry, edited by L. Avramov et al., Math. Sci. Res. Inst. Publ. 51, Cambridge University Press, New York, 2004.

[Brieskorn and Knörrer 1986] E. Brieskorn and H. Knörrer, Plane algebraic curves, Birkhäuser, Basel, 1986. German original, Birkhäuser, 1981.

[De Concini and Procesi 1983] C. De Concini and C. Procesi, "Complete symmetric varieties", pp. 1-44 in Invariant theory (Montecatini, 1982), edited by F. Gherardelli, Lecture Notes in Math. 996, Springer, Berlin, 1983.

[De Concini and Procesi 1985] C. De Concini and C. Procesi, "Complete symmetric varieties, II: Intersection theory", pp. 481-513 in Algebraic groups and related topics (Kyoto/Nagoya, 1983), edited by R. Hotta, Adv. Stud. Pure Math. 6, Kinokuniya, Tokyo, 1985.

[Eisenbud and Sidman 2004] D. Eisenbud and J. Sidman, "The geometry of syzygies", pp. 115-152 in Trends in algebraic geometry, edited by L. Avramov et al., Math. Sci. Res. Inst. Publ. 51, Cambridge University Press, New York, 2004.

[Eisenbud and Sturmfels 1996] D. Eisenbud and B. Sturmfels, "Binomial ideals", Duke Math. J. 84:1 (1996), 1-45.

[Ewald 1996] G. Ewald, Combinatorial convexity and algebraic geometry, Graduate Texts in Math. 168, Springer, Paris, 1996.

[Fenchel 1929] W. Fenchel, "Über Krümmung und Windung geschlossener Raumkurven", Math. Annalen 101 (1929), 238-252. 
[Gerstenhaber 1964] M. Gerstenhaber, "On the deformation of rings and algebras", Ann. of Math. (2) $\mathbf{7 9}$ (1964), 59-103.

[Gerstenhaber 1966] M. Gerstenhaber, "On the deformation of rings and algebras, II", Ann. of Math. (2) 84 (1966), 1-19.

[Goldin and Teissier 2000] R. Goldin and B. Teissier, "Resolving singularities of plane analytic branches with one toric morphism", pp. 315-340 in Resolution of singularities (Obergurgl, 1997), edited by H. Hauser et al., Progr. Math. 181, Birkhäuser, Basel, 2000.

[González Pérez 2003] P. D. González Pérez, "Toric embedded resolutions of quasiordinary hypersurface singularities", Ann. Inst. Fourier (Grenoble) 53:6 (2003), 1819-1881.

[González Pérez and Teissier 2002] P. D. González Pérez and B. Teissier, "Embedded resolutions of non necessarily normal affine toric varieties", C. R. Math. Acad. Sci. Paris 334:5 (2002), 379-382.

[Gromov 1990] M. Gromov, "Convex sets and Kähler manifolds", pp. 1-38 in Advances in differential geometry and topology, edited by F. Tricerri, World Sci. Publishing, Teaneck, NJ, 1990.

[Grünbaum 1967] B. Grünbaum, Convex polytopes, Pure and Applied Mathematics 16, Wiley/Interscience, New York, 1967. Second edition, Springer, 2003 (GTM 221).

[Hanner and Rådström 1951] O. Hanner and H. Rådström, "A generalization of a theorem of Fenchel", Proc. Amer. Math. Soc. 2 (1951), 589-593.

[Hironaka 1964] H. Hironaka, "Resolution of singularities of an algebraic variety over a field of characteristic zero. I, II", Ann. of Math. (2) 79:1-2 (1964), 109-203, 205-326.

[Hochster 2004] M. Hochster, "Tight closure theory and characteristic $p$ methods", pp. 181-210 in Trends in algebraic geometry, edited by L. Avramov et al., Math. Sci. Res. Inst. Publ. 51, Cambridge University Press, New York, 2004.

[Howald 2001] J. A. Howald, "Multiplier ideals of monomial ideals", Trans. Amer. Math. Soc. 353:7 (2001), 2665-2671.

[Katz 1988] D. Katz, "Note on multiplicity", Proc. Amer. Math. Soc. 104:4 (1988), 1021-1026.

[Kempf et al. 1973] G. Kempf, F. F. Knudsen, D. Mumford, and B. Saint-Donat, Toroidal embeddings, I, Lecture Notes in Math. 339, Springer, Berlin, 1973.

[Khovanskii 1979] A. G. Khovanskii, "Geometry of convex bodies and algebraic geometry", Uspekhi Mat. Nauk 34:4 (1979), 160-161.

[Kleiman and Thorup 1996] S. Kleiman and A. Thorup, "Mixed Buchsbaum-Rim multiplicities", Amer. J. Math. 118:3 (1996), 529-569.

[Kouchnirenko 1976] A. G. Kouchnirenko, "Polyèdres de Newton et nombres de Milnor", Invent. Math. 32:1 (1976), 1-31.

[Lipman and Teissier 1981] J. Lipman and B. Teissier, "Pseudorational local rings and a theorem of Briançon-Skoda about integral closures of ideals", Michigan Math. J. 28:1 (1981), 97-116.

[Merle and Teissier 1980] M. Merle and B. Teissier, "Conditions d'adjonction, d'après Du Val", pp. 229-245 in Séminaire sur les singularités des surfaces (Palaiseau 1976/77), edited by M. Demazure et al., Lecture Notes in Math. 777, Springer, 1980. 
[Northcott and Rees 1954] D. G. Northcott and D. Rees, "Reductions of ideals in local rings", Proc. Cambridge Philos. Soc. 50 (1954), 145-158.

[Oka 1997] M. Oka, Non-degenerate complete intersection singularity, Actualités Mathématiques, Hermann, Paris, 1997.

[Rees 1961] D. Rees, "a-transforms of local rings and a theorem on multiplicities of ideals", Proc. Cambridge Philos. Soc. 57:1 (1961), 8-17.

[Rees 1984] D. Rees, "Generalizations of reductions and mixed multiplicities", J. London Math. Soc. (2) 29:3 (1984), 397-414.

[Rees 1986] D. Rees, "The general extension of a local ring and mixed multiplicities", pp. 339-360 in Algebra, algebraic topology and their interactions (Stockholm, 1983), edited by J.-E. Roos, Lecture Notes in Math. 1183, Springer, Berlin, 1986.

[Rees and Sharp 1978] D. Rees and R. Y. Sharp, "On a theorem of B. Teissier on multiplicities of ideals in local rings", J. London Math. Soc. (2) 18:3 (1978), 449463.

[Skoda and Briançon 1974] H. Skoda and J. Briançon, "Sur la clôture intégrale d'un idéal de germes de fonctions holomorphes en un point de $\mathbf{C}^{n}$ ", C. R. Acad. Sci. Paris Sér. A 278 (1974), 949-951.

[Smith 1873] H. J. S. Smith, "On the higher singularities of plane curves", Proc. London Math. Soc. 6 (1873), 153-182.

[Sturmfels 1996] B. Sturmfels, Gröbner bases and convex polytopes, University Lecture Series 8, Amer. Math. Soc., Providence, 1996.

[Teissier 1973] B. Teissier, "Cycles évanescents, sections planes et conditions de Whitney", pp. 285-362 in Singularités à Cargèse (Cargèse, 1972), Astérisque 7-8, Soc. Math. France, Paris, 1973.

[Teissier 1975] B. Teissier, "Appendice", pp. 145-199 in Le problème des modules pour les branches planes, by O. Zariski, Centre de Mathématiques de l'École Polytechnique, Paris, 1975. Second edition, Hermann, 1986.

[Teissier 1977] B. Teissier, "Sur une inégalité à la Minkowski pour les multiplicités", appendix to D. Eisenbud and H. I. Levine, "An algebraic formula for the degree of a $C^{\infty}$ map germ", Ann. Math. (2) 106:1 (1977), 19-44.

[Teissier 1978] B. Teissier, "On a Minkowski-type inequality for multiplicities. II", pp. 347-361 in C. P. Ramanujam, a tribute, edited by K. G. Ramanathan, Tata Studies in Math. 8, Springer, Berlin, 1978.

[Teissier 1979] B. Teissier, "Du théorème de l'index de Hodge aux inégalités isopérimétriques", C. R. Acad. Sci. Paris Sér. A-B 288:4 (1979), A287-A289.

[Teissier 1988] B. Teissier, "Monômes, volumes et multiplicités", pp. 127-141 in Introduction à la théorie des singularités, II, edited by L. D. Tráng, Travaux en Cours 37, Hermann, Paris, 1988.

[Teissier 2003] B. Teissier, "Valuations, deformations, and toric geometry", pp. 361459 in Valuation theory and its applications (Saskatoon, SK, 1999), vol. 2, edited by F.-V. Kuhlmann et al., Fields Inst. Commun. 33, Amer. Math. Soc., Providence, RI, 2003. 
BERNARD TEISSIER

INSTITUT MATHÉMATIQUE DE JUSSIEU

UMR 7586 DU C.N.R.S.

Équipe "GeOmétrie et Dynamique"

BurEau 8E18

175 Rue du Chevaleret

F 75013 PARIS

FRANCE

teissier@math.jussieu.fr 